\title{
Groups containing locally maximal product-free sets of size 4
}

\author{
C. S. Anabanti
}

Communicated by I. Ya. Subbotin

\begin{abstract}
A BStRACT. Every locally maximal product-free set $S$ in a finite group $G$ satisfies $G=S \cup S S \cup S^{-1} S \cup S S^{-1} \cup \sqrt{S}$, where $S S=\{x y \mid x, y \in S\}, S^{-1} S=\left\{x^{-1} y \mid x, y \in S\right\}, S S^{-1}=\left\{x y^{-1} \mid\right.$ $x, y \in S\}$ and $\sqrt{S}=\left\{x \in G \mid x^{2} \in S\right\}$. To better understand locally maximal product-free sets, Bertram asked whether every locally maximal product-free set $S$ in a finite abelian group satisfy $|\sqrt{S}| \leqslant 2|S|$. This question was recently answered in the negation by the current author. Here, we improve some results on the structures and sizes of finite groups in terms of their locally maximal productfree sets. A consequence of our results is the classification of abelian groups that contain locally maximal product-free sets of size 4, continuing the work of Street, Whitehead, Giudici and Hart on the classification of groups containing locally maximal product-free sets of small sizes. We also obtain partial results on arbitrary groups containing locally maximal product-free sets of size 4 , and conclude with a conjecture on the size 4 problem as well as an open problem on the general case.
\end{abstract}

\section{Introduction}

Let $S$ be a non-empty subset of a finite group $G$. Then $S$ is productfree in $G$ if there is no solution to the equation $x y=z$ for $x, y, z \in S$; equivalently, if $S \cap S S=\varnothing$, where $S S=\{x y \mid x, y \in S\}$. For a finite group $G$, a locally maximal product-free set in $G$ is a product-free subset

2020 MSC: 20D60, 05E15, 11B75.

Key words and phrases: product-free sets, locally maximal, maximal, groups. 
$S$ of $G$ such that given any other product-free set $T$ in $G$ with $S \subseteq T$, then $S=T$. Since every product-free set in a finite group $G$ is contained in a locally maximal product-free set in $G$, we can gain information about product-free sets in a group by studying its locally maximal product-free sets. In connection with Group Ramsey Theory, Street and Whitehead [11] noted that every partition of a finite group $G$ (or in fact, of $G^{*}=$ $G \backslash\{1\})$ into product-free sets can be embedded into a covering by locally maximal product-free sets, and hence to find such partitions, it is useful to understand locally maximal product-free sets. Among other results, they calculated locally maximal product-free sets in groups of small orders, up to 16 in $[11,12]$ as well as a few higher sizes. Giudici and Hart [9] started the classification of finite groups containing small locally maximal product-free sets. They classified finite groups containing locally maximal product-free sets of sizes 1 and 2, as well as some of size 3 . The size 3 problem was resolved in [5]. The reader may see $[1,4,6,11]$ for a concept 'filled groups' studied for locally maximal product-free sets. A locally maximal productfree set in a group $G$ can be characterised as a product-free subset $S$ of $G$ satisfying

$$
G=S \cup S S \cup S^{-1} S \cup S S^{-1} \cup \sqrt{S},
$$

where $S S=\{x y \mid x, y \in S\}, S^{-1} S=\left\{x^{-1} y \mid x, y \in S\right\}, S S^{-1}=\left\{x y^{-1} \mid\right.$ $x, y \in S\}$ and $\sqrt{S}=\left\{x \in G \mid x^{2} \in S\right\}$ (see [9, Lemma 3.1]). Each (locally maximal product-free) set $S$ in a finite group of odd order satisfies $|\sqrt{S}|=|S|$. No such result is known for finite groups of even order in general. To better understand locally maximal product-free sets (LMPFS for short), Bertram [7, p. 41] asked the question: does every locally maximal product-free set $S$ in a finite abelian group satisfy $|\sqrt{S}| \leqslant 2|S|$ ? This question was answered in the negation in [3, pp. 2-3]. The answer shows that we can't rely on $|\sqrt{S}| \leqslant 2|S|$ to obtain a reasonable bound on the order of an arbitrary finite abelian group of even order containing a locally maximal product-free set $S$. The set $S=\left\{x^{2}\right\}$ consisting of the unique involution in the Quaternion group $Q_{8}$ is locally maximal product-free in $Q_{8}$ and satisfies $|\sqrt{S}|=6|S|=\frac{3}{4}\left|Q_{8}\right|$. This shows that relying on $|\sqrt{S}| \leqslant 2|S|$ to obtain a bound on all non-abelian finite groups containing locally maximal product-free sets will be disastrous too. We note that $\sqrt{S}$ cannot always be removable from equation 1 as seen in Remark 3.4(a) of this paper, with the locally maximal product-free subset $S=\left\{x, x^{6}\right\}$ of $G=\left\langle x \mid x^{7}=1\right\rangle \cong C_{7}$. Though not every locally maximal product-free set is a maximal (by cardinality) product-free set, the subset $S$ of $C_{7}$ given here is clearly a maximal by cardinality product-free set. In particular, 
$\left|S \cup S S \cup S S^{-1} \cup S^{-1} S\right|=|S \cup S S|=5$ and $|\sqrt{S}|=2$. Unfortunately, this example shows that the proof of Theorem 3 of [10] is not correct, as the author assumed that every element of a finite group $G$ which is not an element of a maximal product-free subset $S$ of $G$ is either an element of $S S, S S^{-1}$ or $S^{-1} S$. The sizes of $S S, S S^{-1}$ and $S^{-1} S$ were optimised in [10, Theorem 3] that it is difficult to get a counter example of the theorem itself, even though the absence of $\sqrt{S}$ made the proof wrong. We devote this paper to obtaining structures and sizes of finite groups in terms of their locally maximal product-free sets $S$, without necessarily relying on $|\sqrt{S}| \leqslant 2|S|$. Throughout this discussion, $G$ is an arbitrary finite group, except where otherwise stated.

\section{Preliminaries}

Let $S$ and $V$ be subsets of $G$. We define the following:

$$
\begin{gathered}
S V=\{s v \mid s \in S, v \in V\} ; \quad S^{-1}=\left\{s^{-1} \mid s \in S\right\} \\
T(S)=S \cup S S \cup S S^{-1} \cup S^{-1} S ; \quad \sqrt{S}=\left\{x \in G \mid x^{2} \in S\right\} .
\end{gathered}
$$

Lemma 2.1 ([9, Lemma 3.1]). Let $S$ be a product-free set in a group $G$. Then $S$ is locally maximal product-free if and only if $G=T(S) \cup \sqrt{S}$.

Proposition 2.2 ([9, Proposition 3.2]). Let $S$ be a LMPFS in G. Then $\langle S\rangle$ is a normal subgroup of $G$. Furthermore, $G /\langle S\rangle$ is either trivial or an elementary abelian 2-group.

Theorem 2.3 ([9, Theorem 3.4]). If $S$ is a LMPFS in $G$, then $|G| \leqslant$ $2|T(S)| \cdot|\langle S\rangle|$.

Notation 2.4. Let $S \subseteq G$. We define $\hat{S}:=\{s \in S \mid \sqrt{\{s\}} \not \subset\langle S\rangle\}$.

Proposition 2.5 ([9, Proposition 3.6]). Suppose $S$ is a LMPFS in $G$ and that $\langle S\rangle$ is not an elementary abelian 2-group. If $|\hat{S}|=1$, then $|G|=2|\langle S\rangle|$.

Proposition 2.6 ([9, Proposition 3.7]). Suppose $S$ is locally maximal product-free in $G$. Then every element $s$ of $\hat{S}$ has even order. Moreover all odd powers of s lie in $S$.

Proposition 2.7 ([9, Proposition 3.8]). Let $S$ be a LMPFS in $G$. If there exists $s \in S$ and integers $m_{1}, \ldots, m_{t}$ such that $\hat{S}=\left\{s, s^{m_{1}}, \ldots, s^{m_{t}}\right\}$, then $|G|$ divides $4|\langle S\rangle|$. 
Lemma 2.8 ([9, Lemma 3.9]). Suppose $S$ is a locally maximal product-free set in a group $G$. If $S \cap S^{-1}=\varnothing$, then $G=T(S) \cup T(S)^{-1}$.

Corollary 2.9 ([9, Corollary 3.10]). If $S$ is a LMPFS in G such that $S \cap S^{-1}=\varnothing$, then $|G| \leqslant 4|S|^{2}+1$.

We write $D_{2 n}=\left\langle x, y \mid x^{n}=y^{2}=(x y)^{2}=1\right\rangle$ for the finite dihedral group of order $2 n$.

Lemma 2.10 ([6, Lemma 3.10]). There is no locally maximal product-free set of size 4 consisting of at most one involution in a finite dihedral group.

Theorem 2.11 ([6, Theorem 3.11]). Suppose $S$ is a LMPFS of size 4 in a finite dihedral group $G$. Then up to automorphisms of $G$, the possible choices are given as follows:

\begin{tabular}{|c|c|}
\hline$|G|$ & $S$ \\
\hline 8 & $\left\{y, x y, x^{2} y, x^{3} y\right\},\left\{x, x^{3}, y, x^{2} y\right\}$ \\
\hline 10 & $\left\{x^{2}, x^{3}, y, x^{4} y\right\}$ \\
\hline 12 & $\left\{x^{3}, y, x y, x^{2} y\right\},\left\{x^{2}, x^{3}, y, x^{5} y\right\},\left\{x, x^{5}, y, x^{3} y\right\},\left\{x, x^{4}, y, x^{3} y\right\}$ \\
\hline 14 & $\left\{x^{2}, x^{3}, y, x^{6} y\right\}$ \\
\hline 16 & $\left\{x^{2}, x^{3}, y, x^{7} y\right\},\left\{x, x^{6}, y, x^{4} y\right\}$ \\
\hline 18 & $\left\{x^{2}, x^{5}, y, x^{8} y\right\}$ \\
\hline 20 & $\left\{x, x^{8}, y, x^{5} y\right\}$ \\
\hline
\end{tabular}

\section{Main results}

Let $S$ be a locally maximal product-free set in a finite group $G$. If the exponent of $G$ is 2 , then $S^{-1} S=S S=S S^{-1}$ and $\sqrt{S}=\varnothing$; so $G=S \cup S S$. If the exponent of $G$ is 3 , then for $x \in \sqrt{S}$, we have that $x^{2} \in S$, so $x^{4}=x \in S S$, and we conclude that $\sqrt{S} \subseteq S S$. In the light of Equation (1) therefore $G=S \cup S S \cup S^{-1} S \cup S S^{-1}$; whence

$$
|G|=\left|S \cup S S \cup S S^{-1} \cup S^{-1} S\right| \leqslant 3|S|^{2}-|S|+1
$$

since $|S S| \leqslant|S|^{2}$ and $\left|S S^{-1} \cup S^{-1} S\right| \leqslant 2|S|^{2}-2|S|+1$. Now, suppose the exponent of $G$ is 4 . If $S \cap S^{-1}=\varnothing$, then $S$ consists of elements of order 4 only, and as the square roots of elements of order 4 have order 8 , we have that $\sqrt{S}=\varnothing$; thus $G=S \cup S S \cup S^{-1} S \cup S S^{-1}$. Again, $|G| \leqslant 3|S|^{2}-|S|+1$. We begin this study by examining locally maximal product-free sets $S$ with the property that $S \cap S^{-1}=\varnothing$. Lemma 2.8 is that if $S$ is a locally maximal product-free set in a finite group $G$ such that $S \cap S^{-1}=\varnothing$, then $G=S \cup S S \cup S^{-1} S \cup S S^{-1} \cup S^{-1} \cup(S S)^{-1}$. Corollary 2.9 is that 
if $S$ is a locally maximal product-free set in a finite group $G$ such that $S \cap S^{-1}=\varnothing$, then $|G| \leqslant 4|S|^{2}+1$. The first result here (Theorem 3.2 below) improves Lemma 2.8 and Corollary 2.9. For finite groups of odd order, Proposition 3.3 below gives a much tighter and broader result for Corollary 2.9.

Notation 3.1. For a subset $S$ of a group $G$, we write $I(S)$ for the set of all involutions in $S$.

Theorem 3.2. Suppose $S$ is a LMPFS in a finite group $G$ such that $S \cap S^{-1}=\varnothing$. Then $G=S S \cup S^{-1} S \cup S S^{-1} \cup(S S)^{-1}$. Moreover, $|G| \leqslant$ $4|S|^{2}-2|S|-|I(G)|+1$.

Proof. First, $S^{-1} \cap S S^{-1}=\varnothing$; for if $x^{-1}=y z^{-1}$ where $x, y, z \in S$, then $z=x y$, a contradiction. Similarly, $S^{-1} \cap S^{-1} S=\varnothing$. In the light of Equation (1) therefore $S^{-1} \subseteq S S \cup \sqrt{S}$. Let $x^{-1} \in S^{-1}$ be arbitrary. Suppose $x^{-1} \in \sqrt{S}$. Then $x^{-2} \in S$. As $x \in S$, we have that $(x)\left(x^{-2}\right)=x^{-1} \in S S$. So, $S^{-1} \subseteq S S$; whence $S \subseteq(S S)^{-1}$, and $G=S S \cup S^{-1} S \cup S S^{-1} \cup(S S)^{-1}$ follows from Lemma 2.8. Clearly, $|G| \leqslant 4|S|^{2}-2|S|+1$. However, each involution used in obtaining the bound of $|G|$ given as " $|G| \leqslant 4|S|^{2}-$ $2|S|+1$ " is counted at least twice. Now, $x \in S S$ if and only if $x \in(S S)^{-1}$; so all such involutions are counted twice. If $x \in S^{-1} S$, then $x=y^{-1} z$ for some $y, z \in S$. Thus, $x=z^{-1} y$ as well. So $x$ is counted at least twice in $S^{-1} S$. The same applies to $x \in S S^{-1}$. By removing the second count on involutions of $G$, we obtain that $|G| \leqslant 4|S|^{2}-2|S|-|I(G)|+1$.

Proposition 3.3. If $S$ is a locally maximal product-free set of size $k$ in a finite group $G$ of odd order and $0 \leqslant\left|S \cap S^{-1}\right|=l \leqslant k$, then $|G| \leqslant$ $3 k^{2}-l(2 k-1)+1$.

Proof. Suppose $S$ is a LMPFS of size $k$ in a finite group $G$ of odd order and $0 \leqslant\left|S \cap S^{-1}\right|=l \leqslant k$. As each element of $S$ has a unique square root, we have that $|\sqrt{S}|=k$. If $l=0$, then $\left|S S^{-1} \cup S^{-1} S\right| \leqslant 2 k^{2}-2 k+1$ and $|S S| \leqslant k^{2}$; so the result follows from Equation (1). Now, suppose $1 \leqslant\left|S \cap S^{-1}\right|=l \leqslant k$. Let $S=\left\{x_{1}, \cdots, x_{l}, x_{l+1}, \cdots, x_{k}\right\}$ and $S^{-1}=$ $\left\{x_{1}, \cdots, x_{l}, x_{l+1}^{-1}, \cdots, x_{k}^{-1}\right\}$. Then $|S S| \leqslant k^{2}-l+1$.

$$
\begin{aligned}
&\left(S S \cup S S^{-1}\right) \backslash S S \subseteq x_{1}\left\{x_{l+1}^{-1}, \cdots, x_{k}^{-1}\right\} \cup x_{2}\left\{x_{l+1}^{-1}, \cdots, x_{k}^{-1}\right\} \\
& \cup \cdots \cup x_{l}\left\{x_{l+1}^{-1}, \cdots, x_{k}^{-1}\right\} \cup x_{l+1}\left\{x_{l+2}^{-1}, x_{l+3}^{-1}, \cdots, x_{k}^{-1}\right\} \\
& \cup x_{l+2}\left\{x_{l+1}^{-1}, x_{l+3}^{-1}, x_{l+4}^{-1}, \cdots, x_{k}^{-1}\right\} \\
& \cup \cdots \cup x_{k}\left\{x_{l+1}^{-1}, x_{l+2}^{-1}, \cdots, x_{k-1}^{-1}\right\} .
\end{aligned}
$$


Therefore

$$
\left|\left(S S \cup S S^{-1}\right) \backslash S S\right| \leqslant l(k-l)+(k-l)(k-l-1)=(k-l)(k-1) .
$$

Similarly,

$$
\left|\left(S S \cup S^{-1} S\right) \backslash S S\right| \leqslant(k-l)(k-1) .
$$

By Equation (1) therefore $|G| \leqslant 3 k^{2}-l(2 k-1)+1$.

Remark 3.4. (a) The bound of $|G|$ in Proposition 3.3 is tight as it is attained with $(k, l)=(2,2)$ as $S=\left\{x, x^{-1}\right\} \subset G=\left\langle x \mid x^{7}=1\right\rangle \cong C_{7}$ meets it. (b) Theorem 3.2 and Proposition 3.3 point at the need for a bound on the sizes of finite groups of even order containing locally maximal product-free sets $S$ satisfying $S \cap S^{-1} \neq \varnothing$. Such universal bound is hard to obtain (see Question 3.29 at the end of the paper) when $G$ is an arbitrary finite group of even order due to the difficulty in bounding $|\sqrt{S}|$ as we saw an example of a locally maximal product-free subset $S$ of $Q_{8}$ satisfying $|\sqrt{S}|=\frac{3}{4}\left|Q_{8}\right|$; an interested reader may see [2, Proposition 2.1] for a family of groups $G$ for which there exists a locally maximal product-free set $T$ such that $|\sqrt{T}|$ is comparable to $|G|$. However, in the finite abelian case, progress is possible.

In the light of Equation (1), a finite abelian group $G$ containing a locally maximal product-free set $S$ can be characterised as:

$$
G=S \cup S S \cup S S^{-1} \cup \sqrt{S} .
$$

If $|G|$ is odd, then as each element of $G$ has exactly one square root, $|\sqrt{S}|=|S|$. Using

$$
|S S| \leqslant \frac{|S|(|S|+1)}{2} \text { and }\left|S S^{-1}\right| \leqslant|S|^{2}-|S|+1
$$

in equation (3), we obtain that

$$
|G| \leqslant \frac{3|S|^{2}+3|S|+2}{2} .
$$

On the other hand, if $|G|$ is even, then using (4) together with $|\sqrt{S}| \leqslant \frac{|G|}{2}$ yield

$$
|G| \leqslant 3|S|^{2}+|S|+2
$$

We note here that $|\sqrt{S}| \leqslant \frac{|G|}{2}$ follows from the fact that $\sqrt{S}$ is productfree in a finite abelian group $G$ whenever $S$ is product-free, and that a product-free set in $G$ has size at most $\frac{|G|}{2}$. 
Observation 3.5. Let $S$ be a set of size $k$ in a finite abelian group $G$ such that $1 \leqslant\left|S \cap S^{-1}\right|=l \leqslant k$. We define $A(l)$ to be a non-negative integer which is less than or equal to the maximal number of identity 1 's in $\left\{x_{1} x_{1}, \cdots, x_{1} x_{k}\right\} \cup\left\{x_{2} x_{2}, \cdots, x_{2} x_{k}\right\} \cup \cdots \cup\left\{x_{k-1} x_{k-1}, x_{k-1} x_{k}\right\} \cup$ $\left\{x_{k} x_{k}\right\}$. So

$$
|S S| \leqslant \frac{k(k+1)}{2}-A(l)+1 .
$$

Suppose $|G|$ is even. Then

$$
A(l):= \begin{cases}\frac{l+1}{2} & \text { if } l \text { is odd } \\ \frac{l}{2} & \text { if } l \text { is even. }\end{cases}
$$

Whence

$$
|S S| \leqslant \frac{k(k+1)-(l-1)}{2} \text { or } \frac{k(k+1)-(l-2)}{2}
$$

according to whether $l$ is odd or even. Now, suppose $|G|$ is odd. Each element of $G$ has a unique inverse; so $l \geqslant 2$ and even. Thus, $A(l)=\frac{l}{2}$, and we conclude from inequality (7) that

$$
|S S| \leqslant \frac{k(k+1)-l+2}{2} .
$$

Inequality (7) can be used to obtain a better bound for $|G|$ when $S \cap S^{-1} \neq \varnothing$. For instance, if $S$ is a locally maximal product-free set of size $k$ in a finite abelian group $G$ of odd order such that $1 \leqslant\left|S \cap S^{-1}\right|=l \leqslant k$, then $|G| \leqslant \frac{3 k^{2}-l(2 k-1)+(3 k+2)}{2}$, and this result improves inequality (5) for $S \cap S^{-1} \neq \varnothing$. Lemma 3.6 below gives a bound on $|G|$ when $S \cap S^{-1}=\varnothing$.

Lemma 3.6. Suppose $S$ is a locally maximal product-free set in a finite abelian group $G$ such that $S \cap S^{-1}=\varnothing$. Then $G=S S \cup S S^{-1} \cup(S S)^{-1}$. Moreover, $|G| \leqslant 2|S|^{2}-|I(G)|+1$. Also, if $I(G) \subseteq I\left(S S^{-1}\right)$, then $|G| \leqslant$ $2|S|^{2}-2|I(G)|+1$.

Proof. As $S S^{-1}=S^{-1} S$, the part " $G=S S \cup S S^{-1} \cup(S S)^{-1}$ " follows from $G=S S \cup S S^{-1} \cup S^{-1} S \cup(S S)^{-1}$ in Theorem 3.2. As $|S S|=\left|(S S)^{-1}\right| \leqslant$ $\frac{|S|(|S|+1)}{2}$ and $\left|S S^{-1}\right| \leqslant|S|^{2}-|S|+1$, we conclude that $|G| \leqslant 2|S|^{2}+1$. However, each involution that gives rise to the bound on $|G|$ is counted at least twice. Suppose $x$ is an involution of $G$. Now, $x \in S S$ if and only if $x \in(S S)^{-1}$; so all such involutions are counted twice. If $x \in S S^{-1}$, then $x=y z^{-1}$ for some $y, z \in S$. Thus, $x=z y^{-1}$ as well. So $x$ is counted at least twice in $S S^{-1}$. By removing the second count on involutions of $G$, 
we obtain that $|G| \leqslant 2|S|^{2}-|I(G)|+1$. Now, suppose $I(G) \subseteq I\left(S S^{-1}\right)$. Let $x \in S S^{-1}$ be an involution. Then there exist $y, z \in S$ (for $y \neq z$ ) such that $x=y z^{-1}$. Now, $1=x^{2}=y^{2} z^{-2}$; so $y^{2}=z^{2}$. This shows that given each involution $y z^{-1} \in S S^{-1}$, we can find two elements $y^{2}, z^{2} \in S S$ such that $y^{2}=z^{2}$. Thus, we can find a pair of repeated elements $\left(y^{2}, z^{2}\right)$ for $y^{2}, z^{2} \in S S$ such that $y^{2}=z^{2}$. By discarding one element in each pair of the repeated elements of $S S$, we obtain that $|G| \leqslant 2|S|^{2}-2|I(G)|+1$.

The first bound on $|G|$ in Lemma 3.6 is tight as $S=\left\{x, y^{3}, x^{3} y\right\} \subset$ $G=\left\langle x, y \mid x^{4}=1=y^{4}, x y=y x\right\rangle \cong C_{4} \times C_{4}$ meets it.

Remark 3.7. An observation of [3, p. 2] is that if a finite abelian group of order less than or equal to 52 contains a LMPFS $S$ of size 6 or less, then $|\sqrt{S}| \leqslant 2|S|$. This observation, together with Equation (3), Inequalities (2), (5) and (8) and Lemma 3.6 imply that if a finite abelian group $G$ contains a locally maximal product-free set of size 4 , then $|G| \leqslant 32$. The locally maximal product-free sets of size 4 in abelian groups of order up to 32 were checked in GAP [8]. We also used the SmallGroup library in [8] to restrict our search to the abelian groups. If at least two LMPFS of size 4 are found in a certain abelian group $G$, we check whether there is an automorphism of $G$ that takes one to another, and if there is, we display only one such set. In other words, we display only one locally maximal product-free set in each orbit of the action of the automorphism groups of $G$. Our computational results are summarised in Table 1 below. For notations in Table $1, n_{4}$ is the number of locally maximal product-free sets of size 4 in $G$ while $M_{4}$ shows the corresponding sizes of each orbit of the displayed locally maximal product-free sets under the action of automorphism groups of $G$. We take this opportunity to correct a mistake of [11, Table 1], where the authors mentioned that every locally maximal product-free set of size 4 in $C_{14}$ is mapped by an automorphism of $C_{14}$ to either $S_{1}=\left\{x^{2}, x^{5}, x^{9}, x^{13}\right\}$ or $S_{2}=\left\{x^{4}, x^{5}, x^{6}, x^{7}\right\}$. This is not true as Table 1 shows that there are five LMPFS up to automorphisms of $C_{14}$. In particular, as $A u t\left(C_{n}\right)$ is isomorphic to the unit group $C_{n}^{\times}$whose order is $\phi(n)$ (where $\phi(n)$ is Euler's totient function), the automorphisms of $C_{14}$ are maps $\Phi_{i}: x \mapsto x^{i}$ for $x \in C_{14}$ and $i \in\{1,3,5,9,11,13\}$. Therefore, the LMPFS $\left\{x, x^{3}, x^{8}, x^{13}\right\}$ and $\left\{x, x^{4}, x^{7}, x^{12}\right\}$ are mapped by $\Phi_{9}$ and $\Phi_{5}$ respectively into $S_{1}$ and $S_{2}$, and the other three product-free sets in Table 1 (viz. $\left\{x, x^{3}, x^{8}, x^{10}\right\},\left\{x, x^{4}, x^{6}, x^{13}\right\}$ and $\left\{x, x^{6}, x^{8}, x^{13}\right\}$ ) are mapped to neither $S_{1}$ nor $S_{2}$. 
TABLE 1. Finite abelian groups containing LMPFS of size 4.

\begin{tabular}{|c|c|c|c|}
\hline$G$ & $n_{4}$ & LMPFS $S$ of size 4 in $G$ & $M_{4}$ \\
\hline$C_{8}=\left\langle x \mid x^{8}=1\right\rangle$ & 1 & $\left\{x, x^{3}, x^{5}, x^{7}\right\}$ & 1 \\
\hline $\begin{array}{c}C_{4} \times C_{2}=\left\langle x_{1}, x_{2}\right| x_{1}^{4}=1=x_{2}^{2}, \\
\left.x_{1} x_{2}=x_{2} x_{1}\right\rangle\end{array}$ & 3 & $\begin{array}{r}\left\{x_{1}, x_{1}^{3}, x_{2}, x_{1}^{2} x_{2}\right\} \\
\left\{x_{1}, x_{1}^{3}, x_{1} x_{2}, x_{1}^{3} x_{2}\right\}\end{array}$ & $\begin{array}{c}2 \\
1\end{array}$ \\
\hline $\begin{array}{c}C_{2}^{3}=\left\langle x_{1}, x_{2}, x_{3}\right| x_{i}^{2}=1, \\
\left.x_{i} x_{j}=x_{j} x_{i} \text { for } 1 \leqslant i, j \leqslant 3\right\rangle\end{array}$ & 7 & $\left\{x_{1}, x_{2}, x_{3}, x_{1} x_{2} x_{3}\right\}$ & 7 \\
\hline$C_{10}=\left\langle x \mid x^{10}=1\right\rangle$ & 2 & $\left\{x, x^{4}, x^{6}, x^{9}\right\}$ & 2 \\
\hline$C_{11}=\left\langle x \mid x^{11}=1\right\rangle$ & 5 & $\left\{x, x^{3}, x^{8}, x^{10}\right\}$ & 5 \\
\hline$C_{12}=\left\langle x \mid x^{12}=1\right\rangle$ & 9 & $\begin{array}{l}\left\{x, x^{4}, x^{6}, x^{11}\right\}, \\
\left\{x, x^{4}, x^{7}, x^{10}\right\}, \\
\left\{x^{2}, x^{3}, x^{8}, x^{9}\right\}, \\
\left\{x^{2}, x^{3}, x^{9}, x^{10}\right\}\end{array}$ & $\begin{array}{l}4, \\
2, \\
2, \\
1\end{array}$ \\
\hline $\begin{array}{c}C_{6} \times C_{2}=\left\langle x_{1}, x_{2}\right| x_{1}^{6}=1=x_{2}^{2} \\
\left.x_{1} x_{2}=x_{2} x_{1}\right\rangle\end{array}$ & 11 & $\begin{array}{r}\left\{x_{1}, x_{1}^{5}, x_{2}, x_{1}^{3} x_{2}\right\} \\
\left\{x_{1}, x_{2}, x_{1}^{4}, x_{1}^{3} x_{2}\right\} \\
\left\{x_{1}, x_{1} x_{2}, x_{1}^{4}, x_{1}^{4} x_{2}\right\}\end{array}$ & $\begin{array}{l}3, \\
6, \\
2\end{array}$ \\
\hline$C_{13}=\left\langle x \mid x^{13}=1\right\rangle$ & 21 & $\begin{array}{l}\left\{x, x^{3}, x^{5}, x^{12}\right\} \\
\left\{x, x^{3}, x^{10}, x^{12}\right\} \\
\left\{x, x^{5}, x^{8}, x^{12}\right\}\end{array}$ & $\begin{array}{l}12, \\
6, \\
3\end{array}$ \\
\hline$C_{14}=\left\langle x \mid x^{14}=1\right\rangle$ & 27 & $\begin{array}{l}\left\{x, x^{3}, x^{8}, x^{10}\right\}, \\
\left\{x, x^{3}, x^{8}, x^{13}\right\}, \\
\left\{x, x^{4}, x^{6}, x^{13}\right\}, \\
\left\{x, x^{4}, x^{7}, x^{12}\right\}, \\
\left\{x, x^{6}, x^{8}, x^{13}\right\}\end{array}$ & $\begin{array}{l}6, \\
6, \\
6, \\
6, \\
3\end{array}$ \\
\hline$C_{15}=\left\langle x \mid x^{15}=1\right\rangle$ & 16 & $\begin{array}{l}\left\{x, x^{3}, x^{5}, x^{7}\right\} \\
\left\{x, x^{3}, x^{7}, x^{12}\right\}\end{array}$ & $\begin{array}{r}8 \\
8\end{array}$ \\
\hline$C_{16}=\left\langle x \mid x^{16}=1\right\rangle$ & 37 & $\begin{array}{l}\left\{x, x^{3}, x^{10}, x^{12}\right\}, \\
\left\{x, x^{4}, x^{6}, x^{9}\right\}, \\
\left\{x, x^{4}, x^{6}, x^{15}\right\}, \\
\left\{x, x^{4}, x^{9}, x^{14}\right\}, \\
\left\{x, x^{6}, x^{9}, x^{14}\right\}, \\
\left\{x, x^{6}, x^{10}, x^{14}\right\}, \\
\left\{x^{2}, x^{6}, x^{10}, x^{14}\right\}\end{array}$ & $\begin{array}{l}8, \\
4, \\
8, \\
4, \\
4, \\
8, \\
1\end{array}$ \\
\hline $\begin{array}{c}C_{4} \times C_{4}=\left\langle x_{1}, x_{2}\right| x_{1}^{4}=1=x_{2}^{4} \\
\left.x_{1} x_{2}=x_{2} x_{1}\right\rangle\end{array}$ & 6 & $\left\{x_{1}, x_{1}^{3}, x_{2}^{2}, x_{1}^{2} x_{2}^{2}\right\}$ & 6 \\
\hline
\end{tabular}


TABLE 1 , continued

\begin{tabular}{|c|c|c|c|}
\hline$G$ & $n_{4}$ & LMPFS $S$ of size 4 in $G$ & $M_{4}$ \\
\hline $\begin{array}{c}C_{8} \times C_{2}=\left\langle x_{1}, x_{2}\right| x_{1}^{8}=1=x_{2}^{2}, \\
\left.x_{1} x_{2}=x_{2} x_{1}\right\rangle\end{array}$ & 42 & $\begin{aligned} &\left\{x_{1}, x_{2}, x_{1}^{6}, x_{1}^{5} x_{2}\right\}, \\
&\left\{x_{1}, x_{2}, x_{1}^{6}, x_{1}^{4} x_{2}\right\}, \\
&\left\{x_{1}, x_{2}, x_{1}^{2} x_{2}, x_{1}^{5} x_{2}\right\}, \\
&\left\{x_{1}, x_{1} x_{2}, x_{1}^{6}, x_{1}^{6} x_{2}\right\}, \\
&\left\{x_{1}, x_{1}^{6}, x_{1}^{2} x_{2}, x_{1}^{6} x_{2}\right\}, \\
&\left\{x_{2}, x_{1}^{2}, x_{1}^{6}, x_{1}^{4} x_{2}\right\}, \\
&\left\{x_{1}^{2}, x_{1}^{6}, x_{1}^{2} x_{2}, x_{1}^{6} x_{2}\right\}\end{aligned}$ & $\begin{array}{l}8, \\
8, \\
8, \\
8, \\
8, \\
1, \\
1\end{array}$ \\
\hline $\begin{array}{c}C_{4} \times C_{2} \times C_{2}=\left\langle x_{1}, x_{2}, x_{3}\right. \\
x_{1}^{4}=1=x_{2}^{2}, x_{3}^{2}=1 \\
\left.x_{i} x_{j}=x_{j} x_{i} \text { for } 1 \leqslant i, j \leqslant 3\right\rangle\end{array}$ & 4 & $\left\{x_{2}, x_{3}, x_{1}^{2}, x_{1}^{2} x_{2} x_{3}\right\}$ & 4 \\
\hline$C_{17}=\left\langle x \mid x^{17}=1\right\rangle$ & 48 & $\begin{array}{l}\left\{x, x^{3}, x^{8}, x^{13}\right\} \\
\left\{x, x^{3}, x^{8}, x^{14}\right\} \\
\left\{x, x^{3}, x^{11}, x^{13}\right\}\end{array}$ & $\begin{array}{l}16 \\
16 \\
16\end{array}$ \\
\hline$C_{18}=\left\langle x \mid x^{18}=1\right\rangle$ & 54 & $\begin{array}{l}\left\{x, x^{3}, x^{5}, x^{12}\right\}, \\
\left\{x, x^{3}, x^{8}, x^{14}\right\}, \\
\left\{x, x^{3}, x^{9}, x^{14}\right\}, \\
\left\{x, x^{3}, x^{12}, x^{14}\right\}, \\
\left\{x, x^{4}, x^{9}, x^{16}\right\}, \\
\left\{x, x^{4}, x^{10}, x^{17}\right\}, \\
\left\{x, x^{5}, x^{8}, x^{12}\right\}, \\
\left\{x, x^{5}, x^{8}, x^{17}\right\}, \\
\left\{x, x^{6}, x^{9}, x^{16}\right\}\end{array}$ & $\begin{array}{l}6, \\
6, \\
6, \\
6, \\
6, \\
6, \\
6, \\
6, \\
6\end{array}$ \\
\hline $\begin{array}{c}C_{6} \times C_{3}=\left\langle x_{1}, x_{2}\right| x_{1}^{6}=1=x_{2}^{3}, \\
\left.x_{1} x_{2}=x_{2} x_{1}\right\rangle\end{array}$ & 48 & $\begin{array}{l}\left\{x_{1}, x_{1}^{5}, x_{2}, x_{1}^{3} x_{2}\right\} \\
\left\{x_{1}, x_{2}, x_{1}^{5} x_{2}^{2}, x_{1}^{3}\right\}\end{array}$ & $\begin{array}{l}24 \\
24\end{array}$ \\
\hline$C_{19}=\left\langle x \mid x^{19}=1\right\rangle$ & 36 & $\begin{array}{l}\left\{x, x^{3}, x^{5}, x^{13}\right\} \\
\left\{x, x^{4}, x^{6}, x^{9}\right\}\end{array}$ & $\begin{array}{l}18 \\
18\end{array}$ \\
\hline $\begin{array}{c}C_{10} \times C_{2}=\left\langle x_{1}, x_{2}\right| x_{1}^{10}=1=x_{2}^{2}, \\
\left.x_{1} x_{2}=x_{2} x_{1}\right\rangle\end{array}$ & 28 & $\begin{array}{l}\left\{x_{1}, x_{2}, x_{1}^{5} x_{2}, x_{1}^{8}\right\}, \\
\left\{x_{1}, x_{1} x_{2}, x_{1}^{4}, x_{1}^{8} x_{2}\right\}, \\
\left\{x_{1}, x_{1} x_{2}, x_{1}^{8}, x_{1}^{6} x_{2}\right\}\end{array}$ & $\begin{array}{c}12 \\
12 \\
4\end{array}$ \\
\hline$C_{20}=\left\langle x \mid x^{20}=1\right\rangle$ & 36 & $\begin{array}{l}\left\{x, x^{3}, x^{10}, x^{16}\right\}, \\
\left\{x, x^{3}, x^{14}, x^{16}\right\}, \\
\left\{x, x^{4}, x^{11}, x^{18}\right\}, \\
\left\{x, x^{5}, x^{14}, x^{18}\right\}, \\
\left\{x, x^{6}, x^{8}, x^{11}\right\}, \\
\left\{x^{2}, x^{5}, x^{15}, x^{16}\right\}\end{array}$ & $\begin{array}{l}8, \\
8, \\
4, \\
8, \\
4, \\
4\end{array}$ \\
\hline
\end{tabular}


TABLE 1, continued

\begin{tabular}{|c|c|c|c|}
\hline$G$ & $n_{4}$ & LMPFS $S$ of size 4 in $G$ & $M_{4}$ \\
\hline$C_{21}=\left\langle x \mid x^{21}=1\right\rangle$ & 34 & $\begin{array}{l}\left\{x, x^{3}, x^{5}, x^{15}\right\}, \\
\left\{x, x^{4}, x^{10}, x^{17}\right\}, \\
\left\{x, x^{4}, x^{14}, x^{16}\right\}, \\
\left\{x, x^{8}, x^{12}, x^{18}\right\}\end{array}$ & $\begin{array}{c}12, \\
12, \\
4, \\
6\end{array}$ \\
\hline$C_{22}=\left\langle x \mid x^{22}=1\right\rangle$ & 10 & $\left\{x, x^{4}, x^{10}, x^{17}\right\}$ & 10 \\
\hline$C_{24}=\left\langle x \mid x^{24}=1\right\rangle$ & 4 & $\left\{x, x^{6}, x^{17}, x^{21}\right\}$ & 4 \\
\hline $\begin{array}{c}C_{9} \times C_{3}=\left\langle x_{1}, x_{2}\right| x_{1}^{9}=1=x_{3}^{2}, \\
\left.x_{1} x_{2}=x_{2} x_{1}\right\rangle\end{array}$ & 36 & $\begin{array}{l}\left\{x_{1}, x_{1} x_{2}, x_{1}^{3}, x_{1}^{7} x_{2}^{2}\right\} \\
\left\{x_{1}, x_{1} x_{2}, x_{1}^{6}, x_{1}^{7} x_{2}^{2}\right\}\end{array}$ & $\begin{array}{l}18 \\
18\end{array}$ \\
\hline $\begin{array}{c}C_{3}^{3}=\left\langle x_{1}, x_{2}, x_{3}\right| x_{i}^{3}=1, \\
\left.x_{i} x_{j}=x_{j} x_{i} \text { for } 1 \leqslant i, j \leqslant 3\right\rangle\end{array}$ & 468 & $\left\{x_{1}, x_{2}, x_{3}, x_{1}^{2} x_{2}^{2} x_{3}^{2}\right\}$ & 468 \\
\hline
\end{tabular}

Suppose a finite nonabelian group $G$ contains a locally maximal product-free set $S$ of size 4. If $|G|$ is odd, then Proposition 3.3 tells us that $|G| \leqslant 49$. Now, suppose $|G|$ is even. If $S \cap S^{-1}=\varnothing$, then Theorem 3.2 tells us that $|G| \leqslant 56$. So the only case left is to bound the size of a finite group $G$ of even order which contains a locally maximal product-free set of size 4 such that $\left|S \cap S^{-1}\right| \geqslant 1$. We use GAP [8] to check all locally maximal product-free sets of size 4 in nonabelian groups of order at most 56 . The result shows that 45 nonabelian groups contains locally maximal product-free sets of size 4 , and over $15 \%$ of them are dihedral groups. More importantly, the largest size of such group is 40 . We shall study a special case for the generating set of the locally maximal product-free sets as we aim to prove the following:

Theorem 3.8. If $G$ is a finite group containing a locally maximal productfree set of size 4 such that every 2 -element subset of $S$ generates $\langle S\rangle$, then $|G| \leqslant 40$.

We develop preliminary results that we shall put together to prove Theorem 3.8. In particular, we aim to prove Theorem 3.8 by considering each of the following three cases: (a) $S$ contains at least two involutions; (b) $S$ contains no involution; (c) $S$ contains only one involution.

Before we proceed, we state the following result (Lemma 3.9 below) for a finite group $G$ which we shall employ whenever necessary, without necessarily quoting the result.

Lemma 3.9. If $S$ is a LMPFS in a group $G$, then $S$ is locally maximal product-free in $\langle S\rangle$. 
Proof. Suppose $S$ is a LMPFS in a finite group $G$. To show that $S$ is locally maximal product-free in $\langle S\rangle$ suffices to show that $S$ is product-free in $\langle S\rangle$ and $T(S) \cup\left\{g \in\langle S\rangle: g^{2} \in S\right\}=\langle S\rangle$. The first is clear since $\langle S\rangle \subseteq G$ and $S$ is product-free in $G$. For the latter, we first recall that $T(S) \subseteq\langle S\rangle$. Let $g \in\langle S\rangle \backslash T(S)$ be arbitrary. As $g \in G$ and $S$ is locally maximal product-free in $G$, we have that $g^{2} \in S$. Therefore for all $g \in\langle S\rangle$, either $g \in T(S)$ or $g^{2} \in S$; whence $S$ is locally maximal product-free in $\langle S\rangle$.

As a consequence to Theorem 2.11, we give the following result:

Corollary 3.10. No finite group contains a LMPFS S of size 4 such that every two element subset of $S$ generates $\langle S\rangle$, and $S$ contains at least two involutions.

Proof. Suppose a finite group $G$ contains a LMPFS $S$ of size 4 such that every two element subset of $S$ generates $\langle S\rangle$, and $S$ contains at least two involutions. Then $\langle S\rangle$ is dihedral. In the light of Lemma 3.9 and Theorem $2.11,\langle S\rangle$ is one of $D_{8}, D_{10}, D_{12}, D_{14}, D_{16}, D_{18}$ or $D_{20}$. Suppose $\langle S\rangle=D_{8}$. Then $\langle S\rangle=\left\langle y, x^{2} y\right\rangle \cong C_{2} \times C_{2}$; a contradiction. Suppose $\langle S\rangle$ is one of $D_{10}, D_{14}, D_{16}, D_{18}$ or $D_{20}$. Then $S$ contains two rotations; so the group generated by $S$ is also the group generated by such two rotations, which is abelian (in particular, not dihedral); a contradiction. Finally, suppose $\langle S\rangle=D_{12}$. If $S=\left\{x^{3}, y, x y, x^{2} y\right\}$, then $\langle S\rangle=\left\langle x^{3}, y\right\rangle \cong C_{2} \times C_{2} ; \mathrm{a}$ contradiction. If $S$ is any of the other three locally maximal product-free sets in $D_{12}$, then the group generated by any two rotations in such $S$ is not dihedral; a contradiction. Therefore, no such $G$ (respectively $S$ ) exists.

Before we proceed, we give the following result.

TABLE 2. Nonabelian groups (of order up to 40) that contain a LMPFS of size 4 .

\begin{tabular}{|c|c|c|l|}
\hline$G$ & $n_{4}$ & LMPFS $S$ of size 4 in $G$ & $M_{4}$ \\
\hline$D_{8}=\langle x, y| x^{4}=1=y^{2}$, & 3 & $\left\{y, x y, x^{2} y, x^{3} y\right\}$, & 1, \\
$\left.x y=y x^{-1}\right\rangle$ & & $\left\{x, x^{3}, y, x^{2} y\right\}$ & 2 \\
\hline$Q_{8}=\langle x, y| x^{4}=1, x^{2}=y^{2}$, & 3 & $\left\{x, x^{3}, y, x^{2} y\right\}$ & 3 \\
$\left.x y=y x^{-1}\right\rangle$ & & & \\
\hline$D_{10}=\langle x, y| x^{5}=1=y^{2}$, & 10 & $\left\{x^{2}, x^{3}, y, x^{4} y\right\}$ & 10 \\
$\left.x y=y x^{-1}\right\rangle$ & & & \\
\hline$Q_{12}=\langle x, y| x^{6}=1, x^{3}=y^{2}$, & 9 & $\left\{x, x^{5}, y, x^{3} y\right\}$, & 3, \\
$\left.x y=y x^{-1}\right\rangle$ & & $\left\{x, x^{4}, y, x^{3} y\right\}$ & 6 \\
\hline
\end{tabular}


TABLE 2, continued

\begin{tabular}{|c|c|c|c|}
\hline$G$ & $n_{4}$ & LMPFS $S$ of size 4 in $G$ & $M_{4}$ \\
\hline $\begin{aligned} A_{4} & = \\
\langle x, y| x^{3}=y^{2} & \left.=(x y)^{3}=1\right\rangle\end{aligned}$ & 2 & $\left\{x, y x, x^{2} y x, x y\right\}$ & 2 \\
\hline $\begin{array}{c}D_{12}=\langle x, y| x^{6}=1=y^{2}, x y= \\
\left.y x^{-1}\right\rangle\end{array}$ & 27 & $\begin{array}{c}\left\{x^{3}, y, x y, x^{5} y\right\} \\
\left\{x^{2}, x^{3}, y, x^{5} y\right\} \\
\left\{x, x^{5}, y, x^{3} y\right\} \\
\left\{x, x^{4}, y, x^{3} y\right\}\end{array}$ & $\begin{array}{l}6, \\
12, \\
3, \\
6\end{array}$ \\
\hline $\begin{array}{c}D_{14}=\langle x, y| x^{7}=1=y^{2}, x y= \\
\left.y x^{-1}\right\rangle\end{array}$ & 42 & $\left\{x^{2}, x^{3}, y, x^{6} y\right\}$ & 42 \\
\hline $\begin{array}{c}\left(C_{4} \times C_{2}\right) \rtimes_{\alpha} C_{2}=\langle x, y| x^{4}= \\
y^{2}=(x y x)^{2}=\left(y x^{-1}\right)^{4}= \\
\left.\left(y x y x^{-1}\right)^{2}=1\right\rangle\end{array}$ & 2 & $\left\{x^{2}, y,(x y)^{2}, x^{3} y x\right\}$ & 2 \\
\hline $\begin{array}{c}C_{4} \rtimes C_{4}=\langle x, y| x^{4}=y^{4}= \\
x^{-1} y x y=x^{2} y^{-1} x^{2} y= \\
\left.\left(y^{-1} x^{-2} y^{-1}\right)^{2}=1\right\rangle\end{array}$ & 2 & $\left\{x^{2}, y, x^{3} y x, x^{2} y^{2}\right\}$ & 2 \\
\hline $\begin{array}{c}M_{16}=C_{8} \rtimes C_{2}=\langle x, y| x^{8}= \\
\left.1=y^{2}, x y=y x^{5}\right\rangle\end{array}$ & 26 & $\begin{array}{c}\left\{x, x^{4}, x^{7}, y\right\}, \\
\left\{x, x^{6}, y, x^{4} y\right\}, \\
\left\{x^{2}, x^{6}, y, x^{4} y\right\}, \\
\left\{x, x^{6}, x^{2} y, x^{6} y\right\} \\
\left\{x^{2}, x^{6}, x^{2} y, x^{6} y\right\}\end{array}$ & $\begin{array}{l}8, \\
8, \\
1, \\
8, \\
1\end{array}$ \\
\hline $\begin{array}{c}D_{16}=\langle x, y| x^{8}=y^{2}=(x y)^{2}= \\
1\rangle\end{array}$ & 48 & $\begin{array}{l}\left\{x^{2}, x^{3}, y, x^{7} y\right\} \\
\left\{x, x^{6}, y, x^{4} y\right\}\end{array}$ & $\begin{array}{l}32, \\
16\end{array}$ \\
\hline $\begin{array}{c}Q D_{16}=\langle x, y| x^{8}=1= \\
\left.y^{2}, x y=y x^{3}\right\rangle\end{array}$ & 16 & $\begin{array}{r}\left\{x, x^{6}, y, x^{4} y\right\} \\
\left\{x, x^{6}, x^{3} y, x^{7} y\right\}\end{array}$ & $\begin{array}{l}8 \\
8\end{array}$ \\
\hline $\begin{array}{c}Q_{16}=\langle x, y| x^{8}=1, x^{4}= \\
\left.y^{2}, x y=y x^{-1}\right\rangle\end{array}$ & 16 & $\left\{x, x^{6}, y, x^{4} y\right\}$ & 16 \\
\hline $\begin{array}{c}D_{8} * C_{4}=\langle x, y, z| \\
x^{2}=y^{2}=z^{4}=1= \\
\left.z^{-1} x z x=z^{-1} y z y=y z^{2} x y x\right\rangle\end{array}$ & 8 & $\left\{x, y, x y z,(x y)^{2}\right\}$ & 8 \\
\hline $\begin{array}{c}D_{18}=\langle x, y| x^{9}=1=y^{2}, x y= \\
\left.y x^{-1}\right\rangle\end{array}$ & 54 & $\left\{x^{2}, x^{5}, y, x^{8} y\right\}$ & 54 \\
\hline $\begin{array}{c}C_{3} \times S_{3}=\langle x, y, z| x^{2}=y^{3}= \\
z^{3}=(x z)^{2}=y^{-1} x y x= \\
\left.z^{-1} y^{-1} z y=1\right\rangle\end{array}$ & 72 & $\begin{array}{c}\{x, y, x z, y z x\} \\
\{x, y, z, x y z\}, \\
\{x, y, z, y z x\} \\
\left\{x, y, z, x y^{2} z x\right\} \\
\{x, y, x y z, y z x\} \\
\left\{x, z, x y z, x y^{2} z\right\} \\
\left\{x, z, y z x, y^{2} z x\right\}, \\
\left\{y, x y, z, x y^{2} z x\right\}\end{array}$ & $\begin{array}{l}6, \\
12, \\
12, \\
12, \\
6, \\
6, \\
6, \\
12\end{array}$ \\
\hline $\begin{array}{c}\left(C_{3} \times C_{3}\right) \rtimes C_{2}=\langle x, y, z| \\
x^{2}=y^{3}=z^{3}=(x y)^{2}=(x z)^{2}= \\
\left.z^{-1} y^{-1} z y=1\right\rangle\end{array}$ & 144 & $\begin{array}{c}\{y, x, x z, x y x z x\} \\
\{y, x, x z, y z x\}\end{array}$ & $\begin{array}{l}72, \\
72\end{array}$ \\
\hline
\end{tabular}


TABLE 2, continued

\begin{tabular}{|c|c|c|c|}
\hline$G$ & $n_{4}$ & LMPFS $S$ of size 4 in $G$ & $M_{4}$ \\
\hline $\begin{array}{c}Q_{20}=\langle x, y| x^{10}=1, x^{5}=y^{2} \\
\left.x y=y x^{-1}\right\rangle\end{array}$ & 20 & $\left\{x, x^{8}, y, x^{5} y\right\}$ & 20 \\
\hline $\begin{array}{c}\text { Suz }(2)=\langle x, y| x^{5}=1= \\
\left.y^{4}, x y=y x^{2}\right\rangle \text { (the only } \\
\text { non-simple Suzuki group). }\end{array}$ & 40 & $\begin{array}{l}\left\{x, y^{2}, x^{2} y, x y^{3}\right\} \\
\left\{x^{3}, y^{2}, x^{2} y, x y^{3}\right\}\end{array}$ & $\begin{array}{l}20 \\
20\end{array}$ \\
\hline $\begin{array}{c}D_{20}=\langle x, y| x^{10}=1=y^{2}, x y= \\
\left.y x^{-1}\right\rangle\end{array}$ & 20 & $\left\{x, x^{8}, y, x^{5} y\right\}$ & 20 \\
\hline $\begin{array}{c}C_{7} \rtimes C_{3}=\langle x, y| x^{3}=y^{7}= \\
\left.y^{-1} x^{-1} y x y^{-1}=1\right\rangle\end{array}$ & 280 & $\begin{array}{c}\left\{x, y,(x y)^{2} x, x y x\right\}, \\
\left\{x, y,(x y)^{2} x, x^{2} y x^{2}\right\}, \\
\left\{x, y,(y x)^{2}, x(x y)^{2}\right\}, \\
\left\{x, y, x y x, x(x y)^{2}\right\}, \\
\left\{x, y, x^{2} y x^{2},(x y)^{2}\right\}, \\
\left\{x, y, y x^{2} y,(x y)^{2}\right\}, \\
\left\{x, x^{2} y, x(x y)^{2} x, y x\right\}, \\
\left\{x, x^{2} y, x y x, y x^{2}\right\}, \\
\left\{x^{2}, y,(x y)^{2} x, x y x\right\}\end{array}$ & $\begin{array}{l}42, \\
21, \\
42, \\
42, \\
42, \\
42, \\
14, \\
14, \\
21\end{array}$ \\
\hline $\begin{array}{c}C_{3} \rtimes C_{8}=\langle a, x| x^{8}=a^{3}= \\
\left.x^{-1} \text { axa }=1\right\rangle\end{array}$ & 39 & $\begin{array}{c}\left\{x, a, x^{6}, x^{4} a\right\} \\
\left\{x, x a, x^{6}, x^{4} a x\right\} \\
\left\{a, x^{2}, x^{6}, x^{4} a\right\} \\
\left\{x^{2}, x^{6}, x^{4} a, x^{3} a x\right\}\end{array}$ & $\begin{array}{l}24 \\
12 \\
2 \\
1\end{array}$ \\
\hline $\begin{array}{c}S L(2,3)=\langle x, y| x^{3}=y^{4} \\
=1=y^{-1} x y x y^{-1} x= \\
\left.x^{-1} y^{-1}\left(x^{-1} y\right)^{2}=\left(x^{-1} y^{-1}\right)^{3}\right\rangle\end{array}$ & 72 & $\begin{array}{c}\left\{x, x^{2} y, y^{3}, y x^{2}\right\} \\
\left\{x, x^{2} y, y^{3}, x(x y)^{2} y\right\} \\
\left\{x, y, x^{2} y^{3}, x^{2} y x\right\} \\
\left\{x^{2} y, y, x^{2} y x, x y^{2}\right\}\end{array}$ & $\begin{array}{l}24 \\
24 \\
12 \\
12\end{array}$ \\
\hline $\begin{array}{c}Q_{24}=\langle x, y| x^{12}=1, x^{6}=y^{2} \\
\left.x y=y x^{-1}\right\rangle\end{array}$ & 4 & $\left\{x, x^{6}, x^{8}, x^{11}\right\}$ & 4 \\
\hline $\begin{array}{c}Q_{12} \times C_{2}=\langle x, y, a| x^{4}=y^{2}= \\
a^{3}=x^{-1} \text { axa }=y x^{-1} y x= \\
\left.a^{-1} \text { yay }=1\right\rangle\end{array}$ & 6 & $\begin{array}{c}\left\{y, a, x^{2}, x^{2} y a\right\} \\
\left\{y, x^{2}, x^{2} y a, x y a x\right\}\end{array}$ & $\begin{array}{l}4, \\
2\end{array}$ \\
\hline $\begin{array}{l}B(2,3)=\langle x, y| x^{3}=y^{3}=1 \\
\left.\quad=\left(x^{-1} y^{-1}\right)^{3}=\left(y^{-1} x\right)^{3}\right\rangle\end{array}$ & 252 & $\begin{array}{l}\left\{y, x,(x y)^{2}, x^{2} y^{2} x y\right\} \\
\left\{y, x,(x y)^{2} y, x^{2} y^{2} x\right\}\end{array}$ & $\begin{array}{l}144, \\
108\end{array}$ \\
\hline $\begin{array}{c}C_{9} \rtimes C_{3}=\langle x, y| x^{9}=1=y^{3} \\
\left.x y=y x^{4}\right\rangle\end{array}$ & 144 & $\begin{array}{c}\left\{x^{6}, x^{8}, y, x^{4} y^{2}\right\} \\
\left\{x^{3}, y^{2}, x^{4} y^{2}, x^{8} y^{2}\right\} \\
\left\{x, x^{3}, x y, x^{4} y^{2}\right\} \\
\left\{x, x^{6}, x y, x^{4} y^{2}\right\}\end{array}$ & $\begin{array}{l}54 \\
54 \\
18 \\
18\end{array}$ \\
\hline $\begin{array}{l}C_{7} \rtimes C_{4}=\langle x, z| x^{4}=z^{7}= \\
\left.x^{-1} z x z=\left(x^{-1} z\right)^{2} x^{-2}=1\right\rangle\end{array}$ & 6 & $\left\{z, x^{2}, x^{3} z^{2} x, x^{2} z^{3}\right\}$ & 6 \\
\hline $\begin{array}{c}\left(C_{4} \times C_{2}\right) \rtimes C_{4}=\langle x, y| x^{4}= \\
y^{4}=y^{2} x^{-1} y^{-2} x=x y x^{2} y^{-1} x= \\
y^{2} x^{-1} y^{-2} x=(y x)^{2}\left(y^{-1} x\right)^{2}= \\
\left.\left(y x y^{-1} x^{-1}\right)^{2}=\left(x y x^{-1} y\right)^{2}=1\right\rangle\end{array}$ & 1 & $\left\{x^{2}, y^{2},(x y)^{2}, x^{2}(x y)^{2} y^{2}\right\}$ & 1 \\
\hline
\end{tabular}


TABLE 2 , continued

\begin{tabular}{|c|c|c|c|}
\hline$G$ & $n_{4}$ & LMPFS $S$ of size 4 in $G$ & $M_{4}$ \\
\hline $\begin{array}{c}C_{4} \rtimes C_{8}=\langle x, y| x^{8}=y^{4}= \\
\left.x^{-1} y x y=y^{-1} x^{-1} y^{2} x y^{-1}=1\right\rangle\end{array}$ & 1 & $\left\{x^{6}, x^{2}, x^{4} y^{2}, y^{2}\right\}$ & 1 \\
\hline $\begin{array}{c}C_{8} \rtimes_{\text {Quasidih type }} C_{4}=\langle x, y| \\
\left.x^{8}=1=y^{4}, x y=y x^{3}\right\rangle\end{array}$ & 9 & $\begin{array}{r}\left\{x, x^{6}, y^{2}, x^{4} y^{2}\right\} \\
\left\{x^{2}, x^{6}, y^{2}, x^{4} y^{2}\right\}\end{array}$ & $\begin{array}{l}8, \\
1\end{array}$ \\
\hline $\begin{array}{c}C_{8} \rtimes_{\text {Dih type }} C_{4}=\langle x, y| x^{8}= \\
\left.1=y^{4}, x y=y x^{-1}\right\rangle\end{array}$ & 17 & $\begin{array}{l}\left\{x, x^{6}, y^{2}, x^{5} y^{2}\right\} \\
\left\{x, x^{6}, y^{2}, x^{4} y^{2}\right\} \\
\left\{x, y^{2}, x^{2} y^{2}, x^{5} y^{2}\right\} \\
\left\{x^{2}, x^{6}, y^{2}, x^{4} y^{2}\right\}\end{array}$ & $\begin{array}{l}4, \\
8, \\
4, \\
1\end{array}$ \\
\hline $\begin{array}{c}C_{4} \rtimes D_{8}=\langle x, y| x y^{-2} x^{-1} y^{-2}= \\
x(x y)^{2} x=x^{2}\left(x y^{-1}\right)^{2}= \\
\left.y^{-1} x^{-1} y^{3} x=1\right\rangle\end{array}$ & 9 & $\begin{array}{c}\left\{y, x^{2}, x^{4} y^{2}, x^{6}\right\}, \\
\left\{x^{5} y x, x^{2}, x^{4} y^{2}, x^{6}\right\}, \\
\left\{x^{2}, y^{2}, x^{4} y^{2}, x^{6}\right\}\end{array}$ & $\begin{array}{l}4, \\
4, \\
1\end{array}$ \\
\hline $\begin{array}{c}\left(C_{4} \rtimes C_{4}\right) \times C_{2}=\langle x, y, z| z^{2}= \\
y^{4}=x^{4}=x^{-1} y x y=z x^{-1} z x= \\
1=z y^{-1} z y=x^{2} y^{-1} x^{2} y= \\
\left.\left(y^{-1} x^{-2} y^{-1}\right)^{2}\right\rangle\end{array}$ & 2 & $\left\{x^{2} y^{2} z, z, x^{2}, y^{2}\right\}$ & 2 \\
\hline $\begin{array}{c}C_{4} \times Q_{8}=\langle x, y, z| x^{4}=y^{4}= \\
z^{4}=1=x^{-1} y x y=z^{-1} x^{-1} z x \\
=z^{-1} y^{-1} z y=y^{2} z x^{-2} z= \\
\left.x^{-1} z x^{-1} y^{-2} z=x^{2} y^{-1} x^{2} y\right\rangle\end{array}$ & 2 & $\left\{x^{2}, y^{2}, z, x^{2} y^{2} z\right\}$ & 2 \\
\hline $\begin{array}{c}\left(C_{2} \times Q_{8}\right) \rtimes C_{2}=\langle x, y, z| x^{4}= \\
z^{2}=x y x y^{-1}=y^{2} x^{2}= \\
y x y x^{-1}=z y^{-1} z y=(x z x)^{2} \\
\left.=\left(z x^{-1}\right)^{4}=\left(z x z x^{-1}\right)^{2}=1\right\rangle\end{array}$ & 2 & $\left\{x^{2}, z,(x z)^{2}, x^{3} z x\right\}$ & 2 \\
\hline $\begin{array}{c}C_{2}^{2} \rtimes C_{2}^{3}=\langle x, y, z| x^{4}=y^{4}= \\
z^{2} x^{2}=y^{-1} x^{-1} y x^{-1}= \\
z^{-1} y^{-1} z y=y^{2} x^{-1} y^{2} x= \\
\left.z x y^{2} z x=\left(x^{-1} y^{-2} x^{-1}\right)^{2}=1\right\rangle\end{array}$ & 2 & $\left\{x^{2}, y^{2}, y z, x^{2} y^{3} z\right\}$ & 2 \\
\hline $\begin{array}{c}C_{4} \rtimes Q_{8}=\langle x, y, z| y^{4}=z^{4}= \\
x^{2} y^{2}=y x y x^{-1}=x^{-1} z x z= \\
\left.z^{-1} y^{-1} z y=1\right\rangle\end{array}$ & 4 & $\left\{x^{2}, z, x^{3} z x, x^{2} z^{2}\right\}$ & 4 \\
\hline $\begin{array}{c}C_{2} \times C_{2} \times Q_{8}=\langle x, y, z, a| x^{4}= \\
a^{2}=z^{2}=y^{2} x^{2}=y x y x^{-1}= \\
a x^{-1} a x=a y^{-1} a y=z x^{-1} z x= \\
\left.(a z)^{2}=z y^{-1} z y=1\right\rangle\end{array}$ & 4 & $\left\{x^{2}, z, a, x^{2} z a\right\}$ & 4 \\
\hline $\begin{array}{c}C_{9} \rtimes C_{4}=\langle x, z| x^{4}=z^{9}= \\
\left.x^{-1} z x z=1\right\rangle\end{array}$ & 18 & $\begin{array}{l}\left\{x^{2}, z, x^{2} z^{2}, x z^{3} x\right\} \\
\left\{x^{2}, z, x^{3} z^{2} x, x^{2} z^{4}\right\} \\
\left\{x^{2}, z, x^{3} z^{3} x, x^{2} z^{4}\right\}\end{array}$ & $\begin{array}{l}6 \\
6, \\
6\end{array}$ \\
\hline $\begin{array}{c}\left(C_{3} \times C_{3}\right) \rtimes C_{4}=\langle x, z, a| x^{4}= \\
z^{3}=a^{3}=x^{-1} z x z=x^{-1} a x a= \\
a^{-1} z^{-1} a z=\left(x^{-1} z\right)^{2} x^{-2}= \\
\left.z^{-1} a x^{-1} a z^{-1} x=1\right\rangle\end{array}$ & 24 & $\left\{z, x^{2}, x^{2} a, x z a x\right\}$ & 24 \\
\hline
\end{tabular}


TABLE 2, continued

\begin{tabular}{|c|c|c|c|}
\hline$G$ & $n_{4}$ & LMPFS $S$ of size 4 in $G$ & $M_{4}$ \\
\hline $\begin{array}{c}C_{3} \times A_{4}=\langle x, y, z| x^{3}=y^{3}= \\
z^{2}=1=y^{-1} x^{-1} y x=z y^{-1} z y= \\
\left.(z x)^{3}=\left(x^{-1} z\right)^{3}\right\rangle\end{array}$ & 144 & $\left\{x^{2}, y z x, x^{2} y^{2}, x^{2} z x^{2}\right\}$ & 144 \\
\hline $\begin{array}{c}C_{5} \rtimes_{\text {inverse map }} C_{8}=\langle x, y| \\
\left.x^{8}=1=y^{5}, x y x^{-1}=y^{-1}\right\rangle\end{array}$ & 4 & & \\
\hline$Q_{40}=\langle x, y| x^{20}=1, x^{10}=$ & 8 & $\left\{x^{2}, x^{-2}, y, x^{4} y^{2}\right\}$ & 4 \\
$\left.y^{2}, x y=y x^{-1}\right\rangle$ & & & \\
\hline $\begin{array}{c}Q_{20} \times C_{2}=\langle x, y, z| x^{10}=1 \\
z^{2}, x^{5}=y^{2}, x y=y x^{-1}, y z= \\
z y, x z=z x\rangle\end{array}$ & 8 & $\left\{x^{-2}, y^{2}, z, x z\right\}$ & 8 \\
\hline
\end{tabular}

For notation in Table $2, n_{4}$ is the number of locally maximal productfree sets of size 4 in $G$ while $M_{4}$ shows the corresponding sizes of each orbit of the displayed locally maximal product-free sets under the action of automorphism groups of $G$.

Theorem 3.11. Let $S$ be a locally maximal product-free set of size 4 in a finite group $G$ such that $|G| \leqslant 57$. Then the possibilities for $S$ and $G$ are given in Tables 1 and 2.

Proof. We checked for groups of order from 8 up to 57 that contains locally maximal product-free sets of size 4 in GAP [8]. Then listed all such locally maximal product-free sets $S$ of size 4 up to automorphisms of each such group $G$ in Tables 1 and 2 .

Corollary 3.12. If $S$ is a LMPFS of size 4 in a finite group $G$ of odd order, then both $S$ and $G$ are contained in Tables 1 and 2.

Proof. Follows from Proposition 3.3 and Theorem 3.11.

Lemma 3.13. Let $S$ be a LMPFS of size 4 in a finite group $G$ such that every 2-element subset of $S$ generates $\langle S\rangle$. If $S$ contains no involution, then either $|G| \leqslant 40$ or $\langle S\rangle$ is cyclic.

Proof. If $S \cap S^{-1}=\varnothing$, then by Theorem 3.2, $|G| \leqslant 57$. Theorem 3.11 tells us that $(G, S)$ is one of the possibilities in Tables 1 and 2. In particular, $|G| \leqslant 40$. Suppose $S \cap S^{-1} \neq \varnothing$. Then $S$ contains two elements $a$ and $b$ such that $b=a^{-1}$. As every 2-element subset of $S$ generates $\langle S\rangle$, we have that $\langle S\rangle=\left\langle a, a^{-1}\right\rangle=\langle a\rangle$. So $\langle S\rangle$ is cyclic.

Proposition 3.14. Suppose $S$ is a LMPFS of size 4 in a group $G$. If $\langle S\rangle$ is cyclic, then $|G| \leqslant 40$. 
Proof. As $\langle S\rangle$ is cyclic, in the light of Lemma 3.9 and Remark 3.7, $|\langle S\rangle| \leqslant 24$. Table 1 shows various possibilities for $G$ and $S$. Proposition 2.6 tells us that each element $s$ of $\hat{S}$ has even order; whence if $\langle S\rangle$ is any of the cyclic groups of odd order, then $\hat{S}=\varnothing$ and we conclude that $G=\langle S\rangle$. In the light of Table 1 therefore $|G| \leqslant 21<40$. Suppose $\langle S\rangle$ is cyclic of even order. Consider $\langle S\rangle=C_{8}=\left\langle x \mid x^{8}=1\right\rangle$ and $S=\left\{x, x^{3}, x^{5}, x^{7}\right\}$. If any of $x, x^{3}, x^{5}$ or $x^{7}$ is contained in $\hat{S}$, then $\hat{S}$ consists of power of a single element; by Proposition 2.7, $|G|$ divides 32. If none of $x, x^{3}, x^{5}$ or $x^{7}$ is contained in $\hat{S}$, then $\hat{S}=\varnothing$; so $G=\langle S\rangle$. Consider $\langle S\rangle=C_{10}=\left\langle x \mid x^{10}=1\right\rangle$ and $S=\left\{x, x^{4}, x^{6}, x^{9}\right\}$. As $x^{4}$ and $x^{6}$ have odd order, by Proposition 2.6, $x^{4}, x^{6} \notin \hat{S}$. Clearly, $x, x^{9} \notin \hat{S}$ since $x^{3},\left(x^{9}\right)^{3} \notin S$. Therefore $\hat{S}=\varnothing$; so $G=\langle S\rangle$. Consider $\langle S\rangle=C_{12}=\left\langle x \mid x^{12}=1\right\rangle$. By Table 1, there are four such LMPFS up to automorphisms of $C_{12}$. By Proposition 2.6, $x^{4} \notin \hat{S}$ because it has odd order. Suppose $S=\left\{x, x^{4}, x^{6}, x^{11}\right\}$. By Proposition 2.6, $x, x^{11} \notin \hat{S}$ because $x^{3},\left(x^{11}\right)^{3} \notin S$. So $\hat{S} \leqslant 1$ and we conclude by Proposition 3.6 that $|G| \leqslant 24$. If $S=\left\{x, x^{4}, x^{7}, x^{10}\right\}$, then by Proposition 2.6, $\hat{S}=\varnothing$ since $(x)^{3},\left(x^{7}\right)^{3},\left(x^{10}\right)^{3} \notin S$; so $G=\langle S\rangle$. Now, suppose $S$ is any of $\left\{x^{2}, x^{3}, x^{8}, x^{9}\right\}$ or $\left\{x^{2}, x^{3}, x^{9}, x^{10}\right\}$. In the light of Proposition 2.6, in the first case, $x^{2}, x^{8} \notin \hat{S}$, and in the latter case, $x^{2}, x^{10} \notin \hat{S}$. If none of $x^{3}$ or $x^{9}$ is an element of $\hat{S}$, then $\hat{S}=\varnothing$, and we conclude that $G=\langle S\rangle$. If any of $x^{3}$ or $x^{9}$ is contained in $\hat{S}$, then both are contained in $\hat{S}$; by Proposition 2.7 therefore $|G|$ divides 48 . Suppose $|G|=48$. As $\sqrt{S}$ has only elements of order at least 3, we note that the number of elements of order at least 3 in $G$ is 46. Among all the 47 nonabelian groups of order 48 , only the groups whose GAP ID are [48, 1], [48, 8], [48, 18], [48, 27] and [48, 28] have 46 elements of order at least 3 . We checked each of them for a LMPFS of size 4 , and could not find such. Therefore, if $S$ is any of $\left\{x^{2}, x^{3}, x^{8}, x^{9}\right\}$ or $\left\{x^{2}, x^{3}, x^{9}, x^{10}\right\}$, then $|G| \leqslant 24$. Now, consider $\langle S\rangle=C_{14}=\left\langle x \mid x^{14}=1\right\rangle$. Up to automorphisms of $C_{14}$, the LMPFS of size 4 in $C_{14}$ are $\left\{x, x^{3}, x^{8}, x^{10}\right\}$, $\left\{x, x^{3}, x^{8}, x^{13}\right\},\left\{x, x^{4}, x^{6}, x^{13}\right\},\left\{x, x^{6}, x^{8}, x^{13}\right\}$ and $\left\{x, x^{4}, x^{7}, x^{12}\right\}$. In the light of Proposition 2.6, all elements of order 7 and 14 in the respective sets $S$ do not lie in $\hat{S}$. This means that in the first four cases, $G=\langle S\rangle$. For the latter case, only the involution is a possible element of $\hat{S}$; thus, $|\hat{S}| \leqslant 1$, and we conclude by Proposition 2.5 that $|G| \leqslant 28$. Consider $\langle S\rangle=C_{16}=\left\langle x \mid x^{16}=1\right\rangle$. Up to automorphisms of $C_{16}$, the LMPFS $S$ of size 4 are $\left\{x, x^{3}, x^{10}, x^{12}\right\},\left\{x, x^{4}, x^{6}, x^{9}\right\},\left\{x, x^{4}, x^{6}, x^{15}\right\},\left\{x, x^{4}, x^{9}, x^{14}\right\}$, $\left\{x, x^{6}, x^{9}, x^{14}\right\},\left\{x, x^{6}, x^{10}, x^{14}\right\}$ and $\left\{x^{2}, x^{6}, x^{10}, x^{14}\right\}$. For the first six cases, $\hat{S}=\varnothing$; so $G=\langle S\rangle$. For the last case, $S=S^{-1}$; so $\langle S\rangle \cong C_{8}$; a contradiction as $\langle S\rangle \cong C_{16}$. Consider $\langle S\rangle=C_{18}=\left\langle x \mid x^{18}=1\right\rangle$. By Table 1 , there are 9 such LMPFS up to automorphisms of $C_{18}$. In the 
light of Proposition 2.6, any of the 9 locally maximal product-free sets $S$ which does not contain the unique involution $x^{9}$ gives rise to $\hat{S}=\varnothing$; so $G=\langle S\rangle$. For the LMPFS which contains the unique involution, we have that $|\hat{S}| \leqslant 1$; whence by Proposition 2.5 therefore $|G| \leqslant 36$. Consider $\langle S\rangle=C_{20}=\left\langle x \mid x^{20}=1\right\rangle$. Here, there are six such LMPFS up to automorphisms of $C_{20}$. They are $\left\{x, x^{3}, x^{14}, x^{16}\right\},\left\{x, x^{4}, x^{11}, x^{18}\right\},\left\{x, x^{5}, x^{14}, x^{18}\right\}$, $\left\{x, x^{6}, x^{8}, x^{11}\right\},\left\{x, x^{3}, x^{10}, x^{16}\right\}$ and $\left\{x^{2}, x^{5}, x^{15}, x^{16}\right\}$. The first four cases can be handled with Proposition 2.6 to give that $\hat{S}=\varnothing$; so $G=\langle S\rangle$. For $S=\left\{x, x^{3}, x^{10}, x^{16}\right\}$, we have that $|\hat{S}| \leqslant 1$; so $|G| \leqslant 40$. Finally, let $S=\left\{x^{2}, x^{5}, x^{15}, x^{16}\right\}$. By Proposition 2.6, the only possible element of $\hat{S}$ are $x^{5}$ and $x^{15}$. If none of them are in $\hat{S}$, then $\hat{S}=\varnothing$ and $G=\langle S\rangle$. Suppose at least one of them is in $\hat{S}$, then as all odd powers of such element lies in $S$, both elements must belong to $\hat{S}$, and Proposition 2.7 tells us that $|G|$ divides 80 . Suppose $|G|=80$. As $\sqrt{S}$ has only elements of order at least 4 , we note that the number of elements of order at least 4 in $G$ is 78 . Among all the 47 nonabelian groups of order 80, only the groups whose GAP ID are $[80,1],[80,3],[80,8],[80,18]$ and $[80,27]$ contain 78 elements of order at least 4 . We checked each of them for a LMPFS of size 4, and couldn't find such. Therefore, if $S=\left\{x^{2}, x^{5}, x^{15}, x^{16}\right\}$, then $|G| \leqslant 40$. For $\langle S\rangle=C_{22}$ or $C_{24}$, there is only one such LMPFS up to automorphisms of the respective groups and a direct check using Proposition 2.6 tells us that $\hat{S}=\varnothing$; so $G=\langle S\rangle$.

Corollary 3.15. If $S$ is a LMPFS of size 4 in a finite group $G$ such that every two element subset of $S$ generates $\langle S\rangle$ and $S$ contains no involution, then $|G| \leqslant 40$.

Proof. Follows from Lemma 3.13 and Proposition 3.14.

Proposition 3.16. Suppose $S$ is a LMPFS of size 4 in a finite group $G$ such that every two element subset of $S$ generates $\langle S\rangle$ and $S$ contains only one involution. Then either $|G| \leqslant 40$ or $S=\{a, b, c, d\}$, where $c$ is the unique involution in $S$ and either $a, b$ and $d$ have order 3 , or that a has order greater than 3 together with $a^{-1}=b d$ and none of $b$ and $d$ is an involution.

Proof. Suppose $S=\{a, b, c, d\}$, where $c$ is an involution, and each of $a, b$ and $d$ has order at least 3. Consider $a^{-1}$. Recall that $G=T(S) \cup \sqrt{S}$. Suppose $a^{-1} \in \sqrt{S}$. Then $a^{-2} \in S$. This implies that either $a$ has order 3 (by $a^{-2}=a$ ) or $\langle S\rangle$ is cyclic (by $a^{-2} \in\{b, c, d\}$; for instance $a^{-2}=b$ 
implies that $\langle a, b\rangle=\langle a\rangle)$. In the latter case, Proposition 3.14 tells us that $|G| \leqslant 40$. Suppose $a^{-1} \in T(S)$. Then

$$
T(S) \subseteq\left\{\begin{array}{c}
1, a, b, c, d, a^{2}, b^{2}, d^{2}, a b, b a, a c, c a, a d, d a, b c, c b, b d, d b, c d, d c, \\
a b^{-1}, b a^{-1}, c a^{-1}, a^{-1} c, c b^{-1}, b^{-1} c, a^{-1} b, b^{-1} a, a d^{-1}, d^{-1} a, \\
d a^{-1}, a^{-1} d, b d^{-1}, d^{-1} b, c d^{-1}, d^{-1} c, d b^{-1}, b^{-1} d
\end{array}\right\}
$$

If

$$
\begin{gathered}
a^{-1} \in\left\{b, b^{2}, d, d^{2}, a b, b a, a d, d a, a b^{-1}, b a^{-1}, a d^{-1}, d a^{-1}, a^{-1} b,\right. \\
\left.b^{-1} a, a^{-1} d, d^{-1} a\right\}
\end{gathered}
$$

then $\langle S\rangle$ is cyclic, generated by either $a, b$ or $d$. In the light of Proposition 3.14 therefore $|G| \leqslant 40$. If $a^{-1} \in\left\{c, a c, c a, a^{-1} c, c a^{-1}\right\}$, then $\langle S\rangle$ is cyclic; again $|G| \leqslant 40$. Since $a$ has order at least 3 , we have that $a^{-1} \notin\{1, a\}$. Note that $a^{-1} \notin\left\{b c, c b, d c, c d, b^{-1} c, c b^{-1}, d^{-1} c, c d^{-1}, b^{-1} d, d b^{-1}, d^{-1} b, b d^{-1}\right\}$; otherwise $S$ is not product-free. The only remaining possibilities is that $a^{-1} \in\left\{a^{2}, b d, d b\right\}$. We also perform a similiar analysis with $b^{-1}$ and $d^{-1}$. Our conclusion is that either $\langle S\rangle$ is cyclic (which by Proposition 3.14 implies that $|G| \leqslant 40$ ) or either $a$ has order 3 with either $a^{-1}=b d$ or $a^{-1}=d b$ with similar statement for $b$ and $d$. In the latter case, we can assume without loss of generality that either all of $a, b$ and $d$ have order 3 , or that $a$ has order greater than 3 together with $a^{-1}=b d$ and none of $b$ and $d$ is an involution.

In the latter part of Proposition 3.16, our goal is to show that $|G| \leqslant 40$. We resolve the first case of the latter part of Proposition 3.16 in Lemma 3.20 below, and the second case in Corollary 3.24, Lemma 3.25 and Remark 3.26. We will be considering the following special case: $S$ is a LMPFS of size 4 in a group $G$ such that every two-element subset of $S$ generates $\langle S\rangle$. Furthermore $S=\{a, b, c, d\}$ where $c$ is an involution but none of $a, b$ and $d$ is an involution. We shall impose an additional condition that ' $\langle S\rangle$ is neither abelian nor dihedral' (see Assumption 3.18 below). To do so, we first clear the air with the following:

Lemma 3.17. Let $S$ be a LMPFS of size 4 in a finite group $G$ such that $S$ contains exactly one involution and every two element subset of $S$ generates $\langle S\rangle$. Suppose $\langle S\rangle$ is either abelian or dihedral. Then $\langle S\rangle$ must be abelian and $|G| \leqslant 40$. 
Proof. In the light of Lemmas 3.9 and 2.10, $\langle S\rangle$ cannot be dihedral. So, it must be that $\langle S\rangle$ is abelian. If $\langle S\rangle$ is cyclic, then Proposition 3.14 tells us that $|G| \leqslant 40$. Now, suppose $\langle S\rangle$ is a non-cyclic abelian group. By Remark 3.7 and Table $1,\langle S\rangle$ is one of $C_{4} \times C_{2}, C_{2}^{3}, C_{6} \times C_{2}, C_{4} \times C_{4}, C_{8} \times C_{2}$, $C_{4} \times C_{2} \times C_{2}, C_{6} \times C_{3}$ and $C_{10} \times C_{2}$. The LMPFS in the groups $C_{4} \times C_{2}, C_{2}^{3}$, $C_{6} \times C_{2}, C_{4} \times C_{4}, C_{4} \times C_{2} \times C_{2}$ and $C_{10} \times C_{2}$ do not meet the requirement of our defined $S$ in terms of the orders of its elements. In fact, the only possibilities (up to automorphisms of respective group) that satisfy the condition that $S$ has only one element of order 2 and other elements have order at least 3 is that $(\langle S\rangle, S) \in\left\{\left(C_{8} \times C_{2},\left\{x_{1}, x_{2}, x_{1}^{6}, x_{1}^{5} x_{2}\right\}\right),\left(C_{8} \times\right.\right.$ $\left.\left.C_{2},\left\{x_{1}, x_{2}, x_{1}^{2} x_{2}, x_{1}^{5} x_{2}\right\}\right),\left(C_{6} \times C_{3},\left\{x_{1}, x_{2}, x_{1}^{5} x_{2}^{2}, x_{1}^{3}\right\}\right)\right\}$. Proposition 2.6 tells us that elements of $\hat{S}$ have even order, and if $s \in \hat{S}$, then all odd powers of $s$ lies in $S$. In the listed representatives of $S$, we see immediately that if a non-involution $x \in S \subset C_{8} \times C_{2}$, then $x^{3} \notin S$, and if a non-involution $y \in S \subset C_{6} \times C_{3}$, then $y^{5} \notin S$. So in all cases $|\hat{S}| \leqslant 1$. In the light of Proposition 2.5 therefore $|G| \leqslant 2|S|$ in each of the possibilities, from where we deduce that $|G| \leqslant 32$ or 36 according as $\langle S\rangle=C_{8} \times C_{2}$ or $\langle S\rangle=C_{6} \times C_{3}$. However, the only possibility is $(\langle S\rangle, S,|G|)=\left(C_{8} \times C_{2},\left\{x_{1}, x_{2}, x_{1}^{5} x_{2}^{2}, x_{1}^{3}\right\}, 32\right)$ since $\langle S\rangle$ is cyclic in each of the other two cases; for instance if $G=C_{6} \times C_{3}$, then $\langle S\rangle=\left\langle x_{1}, x_{1}^{3}\right\rangle=\left\langle x_{1} \mid x_{1}^{6}=1\right\rangle \cong C_{6}$.

Assumption 3.18. Suppose $S$ is a locally maximal product-free set of size 4 in a finite group $G$ such that every two-element subset of $S$ generates $\langle S\rangle$. Furthermore $S=\{a, b, c, d\}$ where $c$ is an involution but none of $a, b$ or $d$ is an involution, and $\langle S\rangle$ is neither abelian nor dihedral.

Lemma 3.19. Suppose Assumption 3.18 holds. Then

$$
\begin{gathered}
I(G) \subseteq\left\{c, a^{2}, b^{2}, d^{2}, a b, b a, a d, d a, b d, d b, a b^{-1}, b^{-1} a,\right. \\
\left.a d^{-1}, d^{-1} a, b d^{-1}, d^{-1} b\right\} .
\end{gathered}
$$

Proof. The set $I(G)$ consisting of all involutions in $G$ is a subset of $T(S)$, because $G=T(S) \cup \sqrt{S}$ and no element of $\sqrt{S}$ can be an involution. Clearly $1, a, b, d$ are not involutions. Suppose $x \in\left\{a^{ \pm 1}, b^{ \pm 1}, d^{ \pm 1}\right\}$, and $c x$ is an involution. Now $\langle S\rangle=\langle c, x\rangle$ because $\langle S\rangle$ is generated by every 2-element subset of $S$. But $\langle c, x\rangle=\langle c, c x\rangle$, which is dihedral; a contradiction. The case where $x c$ is an involution is the same. So we can eliminate $a c, b c, d c, a^{-1} c, b^{-1} c, d^{-1} c, c a, c b, c d, c a^{-1}, c b^{-1}$ and $c d^{-1}$. This means $I(G) \subseteq\left\{c, a^{2}, b^{2}, d^{2}, a b, b a, a d, d a, b d, d b, a b^{-1}, b a^{-1}\right.$, $\left.a^{-1} b, b^{-1} a, a d^{-1}, d a^{-1}, a^{-1} d, d^{-1} a, b d^{-1}, d b^{-1}, b^{-1} d, d^{-1} b\right\}$. If an element 
$g$ is an involution, then $g^{-1}=g$, so we only need to include one representative from $\left\{g, g^{-1}\right\}$ in the list of possible involutions. This means we need only include one of $a b^{-1}$ and $b a^{-1}$, for example. There are six such pairs, allowing us to remove $b a^{-1}, a^{-1} b, d a^{-1}, a^{-1} d, d b^{-1}$ and $b^{-1} d$ from the list as if they are involutions then they will equal an element that is on the list. Thus $I(G) \subseteq\left\{c, a^{2}, b^{2}, d^{2}, a b, b a, a d, d a, b d, d b, a b^{-1}, b^{-1} a, a d^{-1}\right.$, $\left.d^{-1} a, b d^{-1}, d^{-1} b\right\}$.

Lemma 3.20. Suppose Assumption 3.18 holds. If $a, b$ and $d$ all have order 3 , then $\langle S\rangle \cong A_{4}$ and $|G| \leqslant 24$.

Proof. By Lemma 3.19, and the fact that $a^{2}, b^{2}$ and $d^{2}$ are not involutions, we have

$$
I(G) \subseteq\left\{c, a b, b a, a d, d a, b d, d b, a b^{-1}, b^{-1} a, a d^{-1}, d^{-1} a, b d^{-1}, d^{-1} b\right\} .
$$

If $c$ is the only involution in $G$, then $c$ is central, and hence commutes with $a$. But $\langle S\rangle=\langle a, c\rangle$, which implies $\langle S\rangle$ is abelian, contrary to Assumption 3.18. Therefore there are elements $x, y \in\left\{a^{ \pm 1}, b^{ \pm 1}, d^{ \pm 1}\right\}$ with $y \neq x^{ \pm 1}$, such that $x y$ is an involution. This implies $\langle S\rangle=\left\langle x, y: x^{3}=y^{3}=(x y)^{2}=1\right\rangle$, which is a presentation of $A_{4}$. By Proposition 2.6, any element of $\hat{S}$ must have even order; so $\hat{S} \subseteq\{c\}$. In the light of Proposition 2.5 therefore $|G| \leqslant 24$.

Lemma 3.21. Suppose $S$ is a LMPFS of size 4 in a finite group $G$ such that every two-element subset of $S$ generates $\langle S\rangle$. Furthermore suppose $S=\{a, b, c, d\}$ where $c$ is an involution, $a^{-1}=b d$ has order at least 4 and none of $b$ or $d$ is an involution. Then $G$ has either $1,3,5,7$ or 9 involutions, and $I(G) \subseteq\left\{c, a^{2}, b^{2}, d^{2}, a b^{-1}, b^{-1} a, a d^{-1}, d^{-1} a, b d^{-1}, d^{-1} b\right\}$.

Proof. Since $a^{-1}=b d$, we get $b^{-1}=d a$ and $d^{-1}=a b$. None of these elements can be involutions. Now $o(b d)=o(d b)$ and $o(d a)=o(a d)$ and $o(a b)=o(b a)$. Hence these elements can't be involutions either. The result now follows immediately from Lemma 3.19 and the fact that any group containing involutions has an odd number of them.

Proposition 3.22. Suppose Assumption 3.18 holds. Let $K$ be the centralizer $C_{G}(c)$ of $c$ in $G$, and let $J=K \cap T(S)$. Then $|K| \leqslant 4|J|$, and hence $|G| \leqslant 4|J| \cdot\left|c^{G}\right|$.

Proof. Let $K$ be the centralizer in $G$ of $c$. If $x \in K$ and $x^{2}=a$, then that would imply $\langle S\rangle$ is abelian, because $\langle S\rangle=\langle a, c\rangle=\left\langle x^{2}, c\right\rangle$ and $x$ 
commutes with $c$. Similarly $x$ cannot be a square root of $b$ or $d$. Hence $K \subseteq \sqrt{c} \cup J$. Suppose there exist $x, y \in \sqrt{c} \cap K$ with $x y \notin J$. Let $z \in \sqrt{c} \cap K$. Now $x y, x z$ and $y z$ are elements of $K$ because $K$ is a subgroup. Suppose $x z \notin J$ and $y z \notin J$. Then we have $(y z)^{2}=c$, which implies $z y=c y z$. Similarly $y x=c x y$ and $z x=c x z$. But now $(x y z)^{2}=$ $x y z x y z=x y c x z y z=c x y x z y z=c x c x y z y z=c^{2} x^{2}(y z)^{2}=1$. Therefore $x y z \notin \sqrt{c}$. Thus $x y z \in J$. So either $x z \in J, y z \in J$ or $x y z \in J$. Hence $\sqrt{c} \cap K \subseteq x^{-1} J \cup y^{-1} J \cup(x y)^{-1} J$. Remembering that $K=J \cup(\sqrt{c} \cap K)$ we immediately derive $|K| \leqslant 4|J|$. The remaining possibility is that there do not exist $x, y \in \sqrt{c} \cap K$ with $x y \notin J$. This means either $K=J$ (because $\sqrt{c} \cap K=\varnothing)$, or that there is some $x \in \sqrt{c} \cap K$, but for all $y \in \sqrt{c} \cap K$ we have $x y \in J$. Hence $\sqrt{c} \cap K \subseteq x^{-1} J$. Either way, $|K| \leqslant 2|J|$. Hence $|K| \leqslant 4|J|$ and so $|G| \leqslant 4|J| \cdot\left|c^{G}\right|$.

Lemma 3.23. Suppose Assumption 3.18 holds. Let $K$ be the centralizer $C_{G}(c)$ of $c$ in $G$, and let $J=K \cap T(S)$. Then

$$
J \subseteq\left\{\begin{array}{c}
1, c, a^{2}, b^{2}, d^{2}, b a, a d, d b, a b^{-1}, b a^{-1}, a^{-1} b, b^{-1} a \\
a d^{-1}, d a^{-1}, a^{-1} d, d^{-1} a, b d^{-1}, d b^{-1}, b^{-1} d, d^{-1} b
\end{array}\right\} .
$$

In particular, $|J| \leqslant 20$.

Proof. Since $\langle S\rangle$ is not abelian, $J$ doesn't contain $a, b$ or $d$. Similarly no element of the form $x c$ or $c x$ can be contained in $J$, where $x \in\left\{a^{ \pm 1}, b^{ \pm 1}, d^{ \pm 1}\right\}$ as this would imply the presence in $J$ of either $a, b$ or $d$. Hence we remove these elements from our original list for $T(S)$. The other observation is that since $a^{-1}=b d, b^{-1}=d a$ and $d^{-1}=a b$, these three elements cannot be contained in $J$, because again this would imply the presence in $J$ of $a, b$ or $d$.

Corollary 3.24. Suppose $S$ is a LMPFS of size 4 in a finite group $G$ such that every two-element subset of $S$ generates $\langle S\rangle$. In particular, take $S=\{a, b, c, d\}$ where $c$ is an involution, $a^{-1}=b d$ has order at least 4 and none of $b$ or $d$ is an involution. Furthermore, suppose $\langle S\rangle$ is not dihedral or abelian, then $|G| \leqslant 720$. Moreover, $G$ has $1,3,5,7$ or 9 involutions and $I(G) \subseteq\left\{c, a^{2}, b^{2}, d^{2}, a b^{-1}, b^{-1} a, a d^{-1}, d^{-1} a, b d^{-1}, d^{-1} b\right\}$.

Proof. As $a^{-1}=b d$, by Lemma 3.21 we know $\left|c^{G}\right| \leqslant 9$ and by Lemma 3.23 we have $|J| \leqslant 20$. Hence by Proposition $3.22,|G| \leqslant 4 \times 20 \times 9=720$. The latter fact (that $G$ has 1, 3, 5, 7 or 9 involutions and possible elements of $I(G))$ follows from Lemma 3.21. 
Lemma 3.25. In Corollary 3.24, no LMPFS S exists if $G$ contains either 1 or 9 involutions.

Proof. Let $G$ and $S$ be as defined in Corollary 3.24. If $G$ contains only one involution, then $c$ is central; so $\langle S\rangle=\langle a, c\rangle$ is abelian, contradicting the hypothesis. Suppose $G$ contains exactly 9 involutions. So nine out of the ten likely elements of $I(G)$ listed in Corollary 3.24 are involutions. Note that for any $g, h \in G, o(g h)=o(h g)$. So $a b^{-1}$ is an involution if and only if $b^{-1} a$ is, and so on. Since only one of the above list of ten things is not an involution, it must be the case that all of $a b^{-1}, b^{-1} a$, $a d^{-1}, d^{-1} a, b d^{-1}$ and $d^{-1} b$ are involutions, as is $c$, and exactly two of $a^{2}, b^{2}$ and $d^{2}$ are involutions. Without loss of generality, suppose $a^{2}$ and $b^{2}$ are involutions. Using $a^{-1}=b d$, we get $a d^{-1}=a^{2} b$ and so on; so the nine involutions of $G$ as $c, a^{2}, b^{2}, a b^{-1}, b^{-1} a, a^{2} b, a b a, b a b$ and $a b^{2}$. From $\left(b^{-1} a\right)^{2}=(a b a)^{2}$, we obtain $b^{-1} a=a b a^{2} b^{2}$; whence $a b^{-1} a=a^{2} b a^{2} b^{2}=b$. Now $a b^{-1} a^{-1}=b a^{-2}=b a^{2}$; a contradiction since $o(b)=4$, however $\left(b a^{2}\right)^{2}=1=\left(a^{2} b\right)^{2}$. Thus no such locally maximal product-free set $S$ exists.

Remark 3.26. The aim of this remark is to assert that in Corollary $3.24,|G|<40$ if $G$ contains either 3,5 or 7 involutions. If $G$ has exactly 7 involutions, then at least two of the three pairs $a b^{-1}, b^{-1} a, a d^{-1}$, $d^{-1} a, b d^{-1}, d^{-1} b$ are involutions; so it means we can remove at least 4 elements from the count of $J$. Hence $|J| \leqslant 16$ and $\left|c^{G}\right| \leqslant 7$. By Proposition 3.22 therefore $|G| \leqslant 4 \times 16 \times 7=448$. We show that this case is not easily resolvable like the case where the number of involutions in $G$ is 9 as seen in Lemma 3.25. For instance, if the two pairs $a b^{-1}$, $b^{-1} a, b d^{-1}$ and $d^{-1} b$ are involutions, together three other involutions $c$, $(a b)^{2}$ and $a^{2}$, then $|\langle S\rangle|$ yields different values. As $d^{-1}=a b$, we write $\langle S\rangle=\left\langle a, b \mid\left(a b^{-1}\right)^{2}=\left(a b^{2}\right)^{2}=(b a b)^{2}=a^{4}=(a b)^{4}=b^{n}=1\right\rangle$, where $n$ is the order of $b$; for example if $n=3,4,5$ or 6 , then $\langle S\rangle \cong S_{4}, D_{8}, D_{10}$ or $C_{2} \times S_{4}$ respectively. So we can't make further deductions from $\langle S\rangle$, without knowing at least a dihedral subgroup of $\langle S\rangle$. Now, suppose $G$ contains exactly 5 involutions. Then we can remove at least 2 elements from the count of $J$. So $|J| \leqslant 18$ and $\left|c^{G}\right| \leqslant 5$. Hence $|G| \leqslant 4 \times 18 \times 5=360$. Finally, if $G$ contains exactly 3 involutions, then we can't remove anything from $J$ necessarily; so $|J| \leqslant 20$ and $\left|c^{G}\right| \leqslant 3$. Therefore $|G| \leqslant 4 \times 20 \times 3=240$. In each of these cases, $S=\left\{c, b, d,(b d)^{-1}\right\}$, where $b$ and $d$ are arbitrary elements of order at least three. We checked in GAP for existence of a LMPFS $S$ in the respective groups of even orders. Our result is summarised below. 


\begin{tabular}{|l|c|c|l|}
\hline & $|I(G)|$ & $\left|N A G_{|I(G)|}\right|$ & $\begin{array}{l}\text { GAP I.D of groups G contain- } \\
\text { ing required } S\end{array}$ \\
\hline $8 \leqslant n \leqslant 40$ & $3,5,7$ & 47 & {$[20,3]$ and $[32,14]$} \\
\hline $42 \leqslant n \leqslant 240$ & $3,5,7$ & 1665 & - \\
\hline $242 \leqslant n \leqslant 360$ & 5,7 & 4525 & - \\
\hline $362 \leqslant n \leqslant 448$ & 7 & 3036 & - \\
\hline
\end{tabular}

In the table above, $n$ gives the range of orders of the nonabelian groups of even order tested. We performed four checks in GAP. The first line of result is the outcome of our first check. Our first check was for nonabelian groups of orders from 8 up to 40 that contain either 3,5 or 7 involutions. Only 47 nonabelian groups of even order $n \in[8,40]$ contain either 3,5 or 7 involutions. Among these groups, only in the groups whose GAP IDs are $[20,3]$ and $[32,14]$ that we found our required LMPFS. The group of order 20 mentioned is mainly referred to as the only non-simple Suzuki group; it is denoted by $\operatorname{Suz}(2)=\langle x, y| x^{5}=1=$ $\left.y^{4}, x y=y x^{2}\right\rangle$. There are 40 LMPFS of size 4 in this group (examples are $\left\{x, y^{2}, x^{2} y, x y^{3}\right\}$ and $\left\{x^{3}, y^{2}, x^{2} y, x y^{3}\right\}$ ); each of the 40 LMPFS is of our required form, and under automorphisms of the group, is one of the two mentioned. On the other hand, the group of order 32 mentioned is called the semidihedral group of $C_{8}$ and $C_{4}$ of dihedral type. It has a presentation as $C_{8} \rtimes_{\text {Dih type }} C_{4}=\left\langle x, y \mid x^{8}=1=y^{4}, x y=y x^{-1}\right\rangle$. This group has 17 LMPFS of size 4 ; only 8 of them are of our required form, and up to automorphisms of the group, any LMPFS of our kind is either $\left\{x, x^{6}, y^{2}, x^{5} y^{2}\right\}$ or $\left\{x, y^{2}, x^{2} y^{2}, x^{5} y^{2}\right\}$ (4 belonging to each class). Our second check was for nonabelian groups of even orders from order 42 up till 240 that contain either 3, 5 or 7 involutions. Only 1665 nonabelian groups in that range contain either 3,5 or 7 involutions, and our search shows that no such group contains our desired LMPFS of size 4. The table is now easily understood for the third and fourth check, which are precisely the last two rows of the table.

We now turn back to give a proof of Theorem 3.8.

Proof of Theorem 3.8. Suppose $G$ is a finite group containing a LMPFS of size 4 such that every 2-element subset of $S$ generates $\langle S\rangle$. Corollary 3.10 tells us that no such $S$ exists if $S$ were to contain at least two involutions. If $S$ contains no involution, then Corollary 3.15 tells us that $|G| \leqslant 40$. Finally, if $S$ contains exactly one involution, then Proposition 3.16, Lemma 3.20, Lemma 3.17, Corollary 3.24, Lemma 3.25 and Remark 3.26 yield $|G| \leqslant 40$. 
A deduction from the classification of finite groups containing locally maximal product-free sets of size 4 studied in this paper is the following:

Corollary 3.27. If a finite group $G$ contains a LMPFS $S$ of size 4 , then either $|G| \leqslant 40$ or $G$ is nonabelian, $|G|$ is even, $S \cap S^{-1} \neq \varnothing$ and not every 2-element subset of $S$ generates $\langle S\rangle$.

Proof. Follows from Theorems 3.2, 3.8, 3.11, Remark 3.7 and Corollary 3.12 .

We close the discussion on finite groups containing LMPFS of size 4 with the following:

Conjecture 3.28. If a finite group $G$ contains a LMPFS of size 4, then $|G| \leqslant 40$.

In the light of Theorem 3.2 and Proposition 3.3 as well as some computational investigations, we ask the following question:

Question 3.29. Does there exists a finite group $G$ containing a locally maximal product-free set of size $k$ (for $k \geqslant 4$ ) such that $|G|>2 k(2 k-1)$ ?

We write $n_{k}$ (resp. $m_{k}$ ) for the maximal size of a finite abelian group of even (resp. odd) order containing a LMPFS of size $k$. Some experimental results on finite abelian groups $G$ of even order $n_{k}$ (resp. odd order $m_{k}$ ) containing LMPFS of size $k$ are reported below.

\begin{tabular}{|c|c|c|}
\hline$k$ & $n_{k}$ & $G$ \\
\hline 1 & 4 & $C_{4}$ \\
\hline 2 & 8 & $C_{8}$ and $C_{4} \times C_{2}$ \\
\hline 3 & 16 & $C_{4} \times C_{4}$ \\
\hline 4 & 24 & $C_{24}$ \\
\hline 5 & 36 & $C_{36}, C_{18} \times C_{2}, C_{12} \times C_{3}, C_{6} \times C_{6}$ \\
\hline 6 & 48 & $C_{48}, C_{12} \times C_{4}, C_{12} \times C_{2}^{2}, C_{6} \times C_{2}^{3}$ \\
\hline 7 & 64 & $C_{4}^{3}, C_{8} \times C_{4} \times C_{2}$ \\
\hline
\end{tabular}

\begin{tabular}{|c|c|c|}
\hline$k$ & $m_{k}$ & $G$ \\
\hline 1 & 3 & $C_{3}$ \\
\hline 2 & 7 & $C_{7}$ \\
\hline 3 & 15 & $C_{15}$ \\
\hline 4 & 27 & $C_{3}^{3}, C_{9} \times C_{3}$ \\
\hline 5 & 35 & $C_{35}$ \\
\hline 6 & 45 & $C_{45}, C_{15} \times C_{3}$ \\
\hline 7 & 61 & $C_{61}$ \\
\hline
\end{tabular}

One may be moved by the above result to conjecture that 'if a finite abelian group $G$ of even order contains a LMPFS of size $k$, then $|G| \leqslant(k+1)^{2}$. However, such conjectural statement cannot hold in general as $C_{84}$ contains some locally maximal product-free sets of size 8. On another remark, let $C_{2 n}=\left\langle x \mid x^{2 n}=1\right\rangle$ be the finite cyclic group of order $2 n$, and suppose $S$ is a locally maximal product-free set in a finite cyclic group of even order containing the unique involution in the group. Then $S$ is 
also a locally maximal product-free subset of the finite dicyclic group $Q_{4 n}=\left\langle x, y \mid x^{2 n}=1, x^{n}=y^{2}, x y=y x^{-1}\right\rangle$ of order $4 n$; the reason is because $\left\{x^{i} y \mid 0 \leqslant i \leqslant 2 n-1\right\} \subset \sqrt{x^{n}} \subseteq \sqrt{S}$, and already we know that $\left\{x^{i} \mid 0 \leqslant i \leqslant 2 n-1\right\} \subseteq S \cup S S \cup S S^{-1} \cup \sqrt{S}$. Thus, one may obtain a lower bound on the maximal size of a finite group containing a locally maximal product-free set of size $k$ by using the bound from the dicyclic group counterpart. For instance, the maximal size of a finite cyclic group of even order containing a locally maximal product-free set of size $k$ which contains the unique involution is $4,6,12,20,30$ and 40 for $k=1,2,3,4,5$ and 6 respectively. An example of a locally maximal product-free set containing the unique involution in $C_{4}, C_{6}, C_{12}, C_{20}, C_{30}$ and $C_{40}$ is given respectively as $\left\{x^{2}\right\},\left\{x^{2}, x^{3}\right\},\left\{x^{2}, x^{6}, x^{10}\right\},\left\{x^{4}, x^{7}, x^{9}, x^{10}\right\},\left\{x^{2}, x^{6}, x^{15}, x^{22}, x^{27}\right\}$ and $\left\{x^{3}, x^{8}, x^{20}, x^{29}, x^{33}, x^{39}\right\}$. This tells us that there are locally maximal product-free set(s) of sizes $1,2,3,4,5$ and 6 in $Q_{8}, Q_{12}, Q_{24}, Q_{40}, Q_{60}$ and $Q_{80}$ respectively. So if a finite group $G$ contains a locally maximal product-free set of size $1,2,3,4,5$ or 6 , then $|G| \geqslant 8,12,24,40,60$ or 80 respectively. Experimental results as well as results of $[5,9]$ suggest that the largest size of a finite group containing a LMPFS of size 1, 2, 3, 4, 5 or 6 is $8,16,24,40,64$ or 96 respectively. We take this opportunity to list all finite groups $G$ of expected highest possible size which contain locally maximal product-free sets of size $k$ for $k \in\{1,2,3,4,5\}$.

\begin{tabular}{|c|c|}
\hline$k$ & $G$ \\
\hline 1 & $G_{8}:=\left\langle x, y \mid x^{4}=1, x^{2}=y^{2}, x y=y x^{-1}\right\rangle \cong Q_{8}$ \\
\hline 2 & $G_{16 A}:=\left\langle x, y \mid x^{4}=1=y^{4}, x y=y^{-1} x\right\rangle$ \\
\hline & $G_{16 B}:=\langle x, y, z| x^{4}=1=z^{2}, x^{2}=y^{2}, x y=y x^{-1}, y z=z y, x z=$ \\
& $z x\rangle \cong Q_{8} \times C_{2}$ \\
\hline 3 & $G_{24 A}:=\left\langle x, y \mid x^{12}=1, x^{6}=y^{2}, x y=y x^{-1}\right\rangle \cong Q_{24}$ \\
\hline & $G_{24 B}:=\langle x, y, z| x^{4}=1=z^{3}, x^{2}=y^{2}, x y=y x^{-1}, y z=z y, x z=$ \\
& $z x\rangle \cong Q_{8} \times C_{3}$ \\
\hline 4 & $G_{40 A}:=\left\langle x, y \mid x^{8}=1=y^{5}, x y=y^{-1} x\right\rangle$ \\
\hline & $G_{40 B}:=\left\langle x, y \mid x^{20}=1, x^{10}=y^{2}, x y=y x^{-1}\right\rangle \cong Q_{40}$ \\
\hline & $\begin{array}{c}G_{40 C}:=\langle x, y, z| x^{10}=1=z^{2}, x^{5}=y^{2}, x y=y x^{-1}, y z=z y, x z= \\
z x\rangle \cong Q_{20} \times C_{2}\end{array}$ \\
\hline 5 & $G_{64 A}:=\langle a, b| a^{8}=b^{4}=b^{-2} a^{-1} b^{-2} a=a b^{-1} a^{2} b a=$ \\
& $\left.a^{-1} b^{-1} a b^{-1} a^{-1} b^{-1} a b=1\right\rangle$ \\
\hline &
\end{tabular}




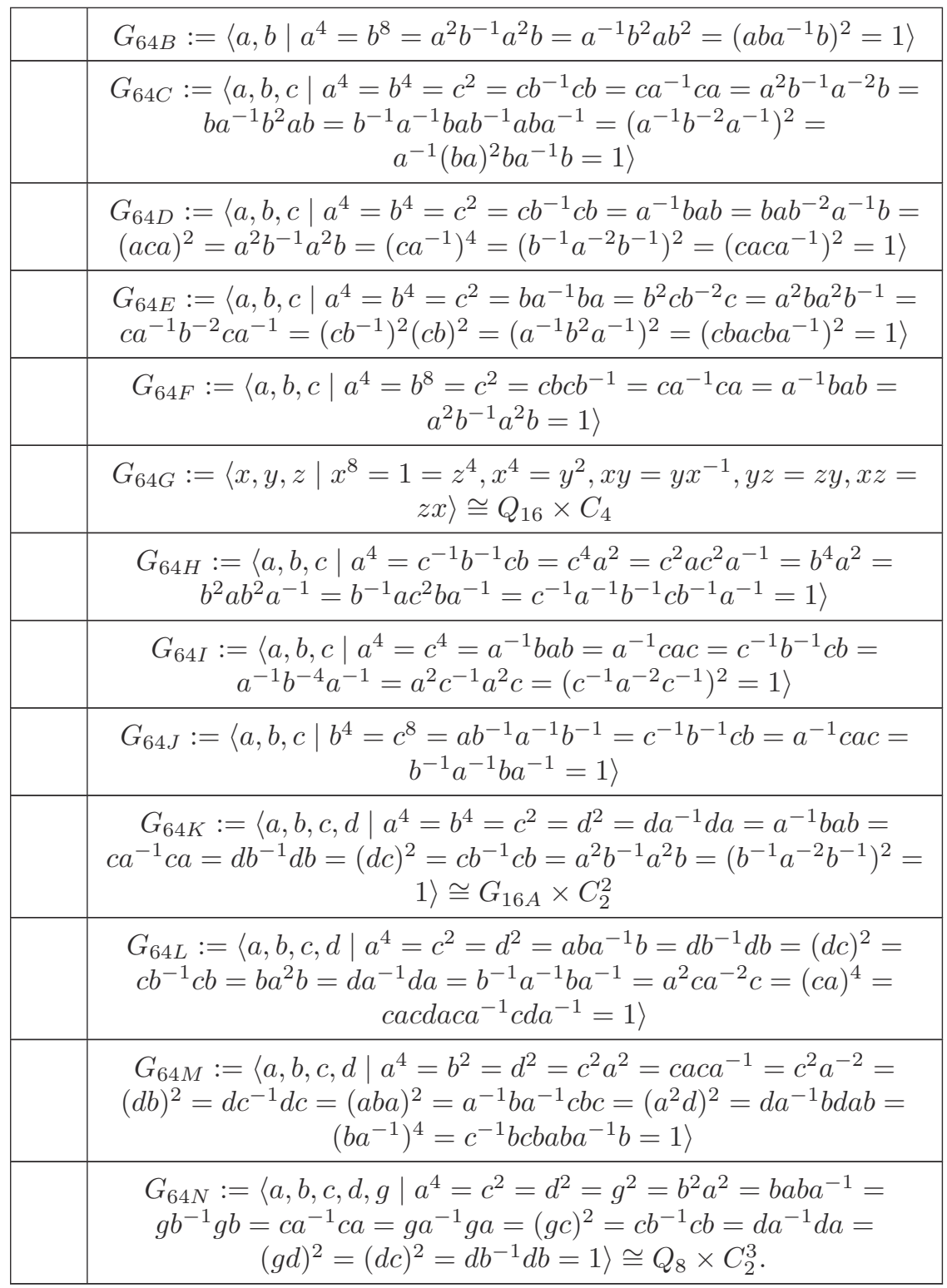

\section{Acknowledgements}

The author is grateful to Professor Sarah Hart for useful discussion during the writing of this paper. He is also thankful to Birkbeck college for the financial support provided. 


\section{References}

[1] C. S. Anabanti, On filled soluble groups, Communications in Algebra, 46(11) (2018), 4914-4917.

[2] C. S. Anabanti, On the three questions of Bertram on locally maximal sum-free sets, Quaestiones Mathematicae, 44(3) (2021), 301-305.

[3] C. S. Anabanti, Three questions of Bertram on locally maximal sum-free sets, Applicable Algebra in Engineering, Communication and Computing, 30(2) (2019), $127-134$.

[4] C. S. Anabanti, G. Erskine and S. B. Hart, Groups whose locally maximal productfree sets are complete, The Australasian Journal of Combinatorics, 71(3) (2018), 544-563.

[5] C. S. Anabanti and S. B. Hart, Groups containing small locally maximal productfree sets, International Journal of Combinatorics, vol. 2016, Article ID 8939182 (2016), 5pp.

[6] C. S. Anabanti and S. B. Hart, On a conjecture of Street and Whitehead on locally maximal product-free sets, The Australasian Journal of Combinatorics, 63(3) (2015), 385-398.

[7] E. A. Bertram, Some applications of Graph Theory to Finite Groups, Discrete Mathematics, 44 (1983), 31-43.

[8] The GAP Group, GAP - Groups, Algorithms, and Programming, Version 4.8.6; 2016, (http://www.gap-system.org).

[9] M. Giudici and S. Hart, Small maximal sum-free sets, The Electronic Journal of Combinatorics, 16 (2009), 17pp.

[10] K. S. Kedlaya, Product-free subsets of groups, American Mathematical Monthly, 105 (1998), 900-906.

[11] A. P. Street and E. G. Whitehead Jr., Group Ramsey Theory, Journal of Combinatorial Theory Series A, 17 (1974), 219-226.

[12] A. P. Street and E. G. Whitehead, Jr., Sum-free sets, difference sets and cyclotomy, Comb. Math., Lect. notes in Mathematics, Springer-Verlag, 403 (1974), 109-124.

\section{CONTACT INFORMATION}

Chimere Department of Mathematics and Applied Mathematics, Stanley University of Pretoria, South Africa

Anabanti E-Mail(s): chimere.anabanti@up.ac.za

Web-page(s): https://www.up.ac.za/

mathematics-and-applied-mathematics/

article/2953786/

dr-cs-chimere-anabanti

Received by the editors: 05.03.2019. 